\title{
On Pure Spinor Superfield Formalism
}

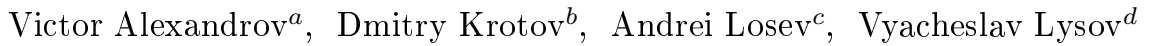 \\ ${ }^{a}$ P.N. Lebedev Physical Institute Theoretical Physics Division Russian Academy of Sciences, \\ ${ }^{b}$ Institute for Nuclear Research of the Russian Academy of Sciences, \\ ${ }^{b, c, d}$ Institute of Theoretical and Experimental Physics, \\ ${ }^{d}$ L.D. Landau Inst. for Theor. Phys. Russian Academy of Sciences , \\ ${ }^{a, b}$ Moscow State University, Department of Physics, \\ ${ }^{d}$ Moscow Institute of Physics and Technology State University,
}

\begin{abstract}
We show that a certain superfield formalism can be used to find an off-shell supersymmetric description for some supersymmetric field theories where conventional superfield formalism does not work. This "new" formalism contains auxiliary variables $\lambda^{\alpha}$ in addition to conventional super-coordinates $\theta^{\alpha}$. The idea of this construction is similar to the pure spinor formalism developed by N.Berkovits. It is demonstrated that using this formalism it is possible to prove that the certain Chern-Simons-like (Witten's OSFT-like) theory can be considered as an off-shell version for some on-shell supersymmetric field theories. We use the simplest nontrivial model found in [2] to illustrate the power of this pure spinor superfield formalism. Then we redo all the calculations for the case of 10-dimensional Super-Yang-Mills theory. The construction of off-shell description for this theory is more subtle in comparison with the model of [2] and requires additional $Z_{2}$ projection. We discover experimentally (through a direct explicit calculation) a non-trivial $Z_{2}$ duality at the level of Feynman diagrams. The nature of this duality requires a better investigation.
\end{abstract}

\section{Introduction}

The importance of the off-shell formulation of supersymmetric field theories is well known. The off-shell SUSYinvariant actions can be found only in limited number of cases for small number of supercharges and in certain space-time dimensions. These formulations are usually based on the superfield formalism. The main advantage of off-shell formulation is the possibility to prove non-renormalization theorems and derive Ward identities on correlation functions. However, in contrast to on-shell formulation, there are auxiliary fields in addition to physical degrees of freedom. The number of these fields may be very large and even infinite.

In the recent paper [2] it was demonstrated that the classical actions for different quantum field theories can be obtained as effective actions from the single fundamental theory of Chern-Simons (or Witten's OSFT) form

$$
S^{\text {Fund }}=\int \operatorname{Tr}\left(<\mathcal{P}, Q_{B} \mathcal{A}>+g<\mathcal{P}, \mathcal{A}^{2}>\right)
$$

Close constructions was originally suggested in [3] and [5]. In the present paper we will demonstrate that all these effective theories are in fact invariant under the global SUSY transformation at least on-shell. The main new results of the present consideration are that action (1) is an off-shell version of all these effective theories and that pure spinor formalism can be considered as a convenient superfield formalism which allows to write the off-shell action in terms of component fields. The definition of the fields, operator $Q_{B}$ and canonical pairing $<,>$ can be found in the section 4 , see also the introduction to [2]. In this previous paper we argued that integrating out some fields from the action (1) one can obtain physically interesting effective action. In the present paper we show that all these fields which are integrated out are nothing but auxiliary fields needed to restore the off-shell invariance of the on-shell supersymmetric effective action. This view on this procedure is very much in the spirit of [6].

The subject of the present paper is the descent of off-shell supersymmetry of the action (1) down to its effective action. To control the SUSY properties of these actions and discuss the descent of symmetry it is convenient to introduce an auxiliary action $S^{S U S Y}$ (interacting with superghosts) defined as

$$
S^{S U S Y}=\int \operatorname{Tr}\left(<\mathcal{P}, Q_{B} \mathcal{A}>+g<\mathcal{P}, \mathcal{A}^{2}>+<\mathcal{P}, \varepsilon Q^{s} \mathcal{A}>+<\mathcal{P}, \eta^{\mu} \partial_{\mu} \mathcal{A}>-\eta_{\mu}^{*}\left(\varepsilon \gamma^{\mu} \varepsilon\right)\right)
$$


The first two terms in this action are exactly those of $S^{F u n d}$. The third and the fourth terms give the algebra of symmetry (SUSY + translations) and the last term is determined by the structure constants of the SUSY algebra $\left\{Q_{\alpha}^{s}, Q_{\beta}^{s}\right\}=2 \gamma_{\alpha \beta}^{\mu} \partial_{\mu}$. Here $\varepsilon^{\alpha}$ and $\eta^{\mu}$ are the ghosts for the global symmetry of $S^{F u n d}\left(\varepsilon^{\alpha}\right.$ - for supersymmetry, $\eta^{\mu}$ - for translations). Hence they do not depend on space-time coordinates. Introduction of these auxiliary fields and addition of the last tree terms into the action (2) is needed to guarantee that action (2) satisfies classical Batalin-Vilkovisky (BV) Master Equation over all the fields including ghosts $\varepsilon$ and $\eta$. This fact is equivalent to the condition that $S^{\text {Fund }}$ (the first two terms in $S^{S U S Y}$ ) is invariant under the algebra of symmetry generated by $Q^{s}$ and $\partial_{\mu}$ and to the condition that this algebra is closed off-shell. As it was mentioned in the section 2 of [2] (for rigorous proof see [4]), integration of BV action over a lagrangian submanifold preserves BV invariance of the effective action. This invariance is what is left from the off-shell invariance of the fundamental action. In particular it leads to the statement that the ghost independent part of the effective action is invariant under the on-shell SUSY transformation.

Thus, the standard ideology of Batalin-Vilkovisky formalism allows us to control how the off-shell symmetry of initial action is inherited in the effective action. Usually BV formalism is used to control gauge symmetries [1]. In this paper we apply the same technique to study the descent of global supersymmetry.

In the section 4 we illustrate these ideas in the rather non-trivial model with 5 quadrics found in [2]. Then in section 5 we apply the same technique to the more interesting model - 10-dimensional Super YangMills. Application of this procedure to SYM is more subtle because after evaluation of effective action on the cohomologies of $Q$-operator one should make a $Z_{2}$ projection identifying the fields $\mathrm{A}$ and $\mathrm{P}$ (representatives of cohomologies in $\mathcal{A}$ and $\mathcal{P}$ ). Remarkably, this projection also preserves BV invariance of the action. At the level of our present understanding this fact seems to be accidental. This observation allows us to find a non-trivial $Z_{2}$ duality at the level of explicit calculation of Feynman diagrams. This duality states that there are certain identities between different Feynman diagrams like

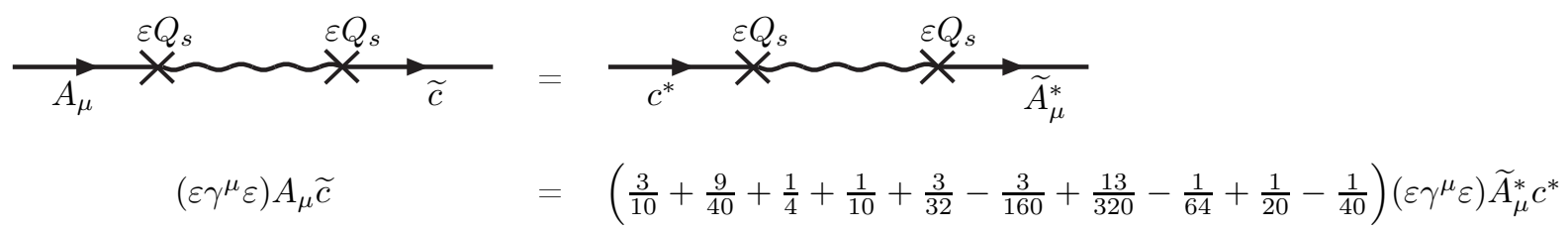

after the identification $\widetilde{c}=c^{*}$ and $\widetilde{A}_{\mu}^{*}=A_{\mu}$. The calculation of the diagram in the 1.h.s. is almost automatic. The calculation of the diagram in the r.h.s. is rather involved. It contains a lot of contributions. Each contribution requires a lot of $\gamma$-matrix algebra including Fiertz identities and different spinor expansions. However, all this contributions collapse in the end to unity which coincides with the 1.h.s. and is an example of the $Z_{2}$ duality. This duality has been checked experimentally for all the diagrams arising in the calculation of effective action. The fundamental nature of this duality is not clear for us. However, what can be said is that the action of SYM with all the SUSY structures satisfies BV equation over all the fields as a consequence of this observed $Z_{2}$ duality of Feynman diagrams.

Summarizing the introduction we would like to list our main results obtained in the present paper.

- It is demonstrated that the Pure Spinor Formalism can be considered as a Superfield Formalism for a large class of interesting quantum field theories - pre-theories (see section 5 for definition).

- It is shown that action (1) can be considered as an off-shell supersymmetric version of these effective pre-theories. The action and degrees of freedom of an effective action are dictated by the choice of the system of quadrics $f^{\mu}(\lambda)$.

- All effective theories obtained after evaluation of effective action of (1) above the cohomologies of $Q$ operator are at least on-shell supersymmetric.

- To obtain the BV version of effective action with the SUSY structures in case of 10-dimensional SYM one should make a $Z_{2}$ projection on the space of fields after the calculation of effective action for (1) (see section 5 for details). For the present moment we do not know whether this $Z_{2}$ symmetry of effective action can be considered as coming from the $Z_{2}$ symmetry of the fundamental action. Would that be possible, we construct the off-shell description of 10 dimensional SYM.

- A non-trivial $Z_{2}$ duality at the level of Feynman diagrams is discovered for the case of $S O(10)$ quadrics $f^{\mu}(\lambda)=\lambda \gamma^{\mu} \lambda$. Evaluation of the diagrams on the one side of this duality is almost automatic. The corresponding calculation on the other side is rather complicated. 
Through the whole paper we use the notation $\gamma_{\alpha \beta}^{\mu}$ to define the system of quadrics as $f^{\mu}(\lambda)=\lambda^{\alpha} \gamma_{\alpha \beta}^{\mu} \lambda^{\beta}$. We would like to stress that we do not restrict ourselves to consider $\gamma_{\alpha \beta}^{\mu}$ as conventional Dirac $\gamma$-matrices. We treat them as a set of constant matrices, symmetric w.r.t. $\alpha$ and $\beta$. As it was shown in [2] this "extension" of the standard Berkovits' construction allows to obtain a zoo of non-trivial effective theories for (1). Only for the case of 10-d SYM $\gamma_{\alpha \beta}^{\mu}$ are conventional $S O(10) \gamma$-matrices.

\subsection{From Off-shell to On-shell Theory Through BV Construction}

First of all we would like to clarify the difference between off-shell and on-shell supersymmetric descriptions of a theory.

\section{Off-shell description.}

Suppose there is an action $S^{c l}$ and a closed algebra generated by $Q_{\alpha}$ and $\partial_{\mu}$, defined by $\left\{Q_{\alpha}, Q_{\beta}\right\}=2 \gamma_{\alpha \beta}^{\mu} \partial_{\mu}$. By off-shell description we mean the following. Action $S^{c l}$ should be invariant under the transformation

$$
\delta_{\epsilon}^{s} S^{c l}=\epsilon^{\alpha} Q_{\alpha} S^{c l}=0, \quad \delta_{\zeta}^{s} S^{c l}=\zeta^{\mu} \partial_{\mu} S^{c l}=0
$$

and that the commutator of two SUSY transformations with parameters $\epsilon_{1}$ and $\epsilon_{2}$, being applied to arbitrary field $A$ from the action $S^{c l}$ should satisfy

$$
\left[\delta_{\epsilon_{1}}, \delta_{\epsilon_{2}}\right] A=2\left(\epsilon_{1} \gamma^{\mu} \epsilon_{2}\right) \partial_{\mu} A
$$

In these formulas $\zeta^{\mu}$ is parameter for translations.

\section{On-shell description}

By on-shell description we mean that action $S^{c l}$ is invariant under the transformation $\delta S^{c l}=0$. However, the commutator of transformations, being applied to a component field $A$ contains corrections proportional to a gauge transformation and to the equations of motion (e.o.m.)for some fields

$$
\left[\delta_{\epsilon_{1}}, \delta_{\epsilon_{2}}\right]=2\left(\epsilon_{1} \gamma^{\mu} \epsilon_{2}\right) \partial_{\mu}+\delta_{\text {gauge }}+R\left(\epsilon_{1}, \epsilon_{2}\right) \text { (e.o.m.) }
$$

Commutator in the 1.h.s. should be applied to component fields. See section 2 and 3 for details.

\section{BV description}

A convenient tool to treat an action and symmetries on the same footing is to use BV formalism. The idea is to add to the classical action $S^{c l}(\varphi)$ (here $\varphi$ denotes all the fields) its symmetries $V_{\alpha}(\varphi)$ with ghosts $\varepsilon^{\alpha}$ (with opposite parity to $\epsilon^{\alpha}$ ) to form BV action.

$$
S^{B V}=S^{c l}+\varepsilon^{\alpha} V_{\alpha}(\varphi) \varphi^{*}+\eta^{\mu} \partial_{\mu} \varphi \varphi^{*}+\left(\varepsilon \gamma^{\mu} \varepsilon\right) \eta_{\mu}^{*} .
$$

The fact that $S^{B V}$ satisfies BV equation is equivalent to the condition that $S^{c l}$ is off-shell symmetric.

The idea is to integrate the action (4) over auxiliary fields. This integration preserve BV invariance. Effective action after integration can be written as

$$
S^{e f f}=S^{c l}+\varepsilon^{\alpha} V_{\alpha}(\varphi) \varphi^{*}+\eta^{\mu} \partial_{\mu} \varphi \varphi^{*}+\left(\varepsilon \gamma^{\mu} \varepsilon\right) \eta_{\mu}^{*}+
$$

$+($ terms quadratic in antifields $)+($ terms responsible for gauge fixing)

Here the set of fields $\varphi$ in the equation (5) is different from the set of fields $\varphi$ in the equation (4). The same is true for the transformations $V_{\alpha}(\varphi)$. Thus action $S^{\text {eff }}$ also satisfies BV equation. This BV action provides on-shell description of initial theory. From the terms written in the second line of (5) one can straightforwardly extract corrections arising in the commutator (3).

Thus our general philosophy can be summarized in the form of the fig. 1 . 


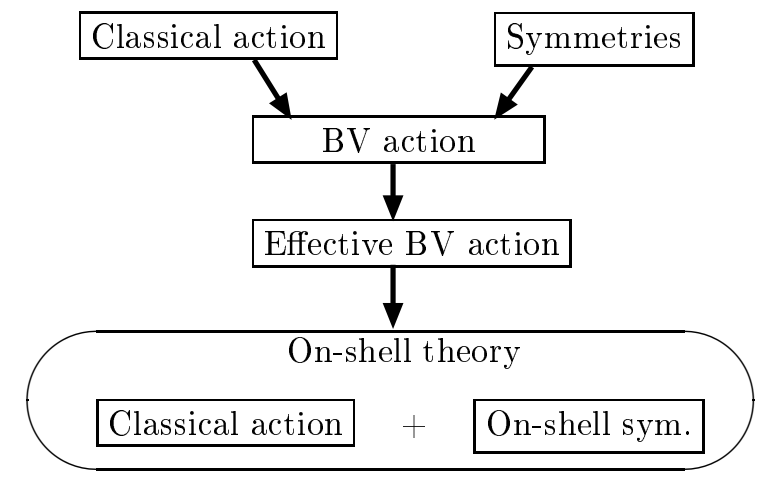

Figure 1: General philosophy

First of all we unite an action and its symmetries in the form of BV action $\left(S^{S U S Y}\right)$, i.e. coupled to superghosts. Then we integrate over lagrangian submanifold to find effective action, having non-standard coupling to ghosts. Finally we extract information about on-shell theory from this effective BV action.

\section{Quantum Mechanics}

We start from the simplest example - supersymmetric quantum mechanics. One can write the off-shell SUSYinvariant action (7) by introducing auxiliary field $D$. Integrating out this field one can obtain the action which contains only physical degrees of freedom, but is no longer off-shell invariant. This means that the algebra of SUSY-transformation can be closed only on-shell.

The algebra of supersymmetry is given by

$$
\begin{array}{r}
\{Q, \bar{Q}\}=2 i \partial_{t} \\
Q^{2}=\bar{Q}^{2}=0
\end{array}
$$

its representation in superspace is

$$
\begin{aligned}
& Q=\partial_{\theta}+i \bar{\theta} \partial_{t} \\
& \bar{Q}=\partial_{\bar{\theta}}+i \theta \partial_{t}
\end{aligned}
$$

and the general superfield is given by

$$
\Phi=x+\theta \bar{\psi}+\bar{\theta} \psi+\theta \bar{\theta} D
$$

The question which we discuss in the present section is: How can the on-shell SUSY invariance be described using BV language?

The off-shell SUSY invariant action for quantum mechanics can be written as:

$$
S^{Q M}=\int d t\left(\frac{1}{2}\left(\partial_{t} x\right)^{2}-i \bar{\psi} \partial_{t} \psi+\frac{1}{2} D^{2}-W(x)^{\prime} D-W(x)^{\prime \prime} \psi \bar{\psi}\right)
$$

Here $W(\Phi)$ stands for the superpotential. The transformations of the component fields $\delta \Phi=(\varepsilon Q+\overline{\varepsilon Q}) \Phi$ are

$$
\begin{array}{r}
\delta x=\epsilon \bar{\psi}+\bar{\epsilon} \psi \\
\delta \psi=-\epsilon\left(i \partial_{t} x+D\right) \\
\delta \bar{\psi}=\bar{\epsilon}\left(-i \partial_{t} x+D\right) \\
\delta D=i \bar{\epsilon} \partial_{t} \psi-i \epsilon \partial_{t} \bar{\psi}
\end{array}
$$

Using these expressions it is straightforward to calculate the commutator of two supersymmetry transformations

$$
\left[\delta_{1}, \delta_{2}\right] \psi=2 i\left(\bar{\epsilon}_{1} \epsilon_{2}-\bar{\epsilon}_{2} \epsilon_{1}\right) \partial_{t} \psi
$$

This result is consistent with the algebra (6), which states that anticommutator of SUSY-charges is proportional to the shift transformation. Now we integrate upon the auxiliary field $D$, substituting $D=W^{\prime}(x)$. Conducting similar computations for the commutator one can find

$$
\begin{array}{r}
{\left[\delta_{1}, \delta_{2}\right] \psi=2 i\left(\bar{\epsilon}_{1} \epsilon_{2}-\bar{\epsilon}_{2} \epsilon_{1}\right) \partial_{t} \psi-} \\
-\left(\bar{\epsilon}_{1} \epsilon_{2}-\bar{\epsilon}_{2} \epsilon_{1}\right)\left(i \partial_{t} \psi-W^{\prime \prime} \psi\right)+2 \epsilon_{1} \epsilon_{2}\left(i \partial_{t} \bar{\psi}+W^{\prime \prime} \bar{\psi}\right)
\end{array}
$$


The terms in the second line are proportional to the equations of motion for the fermions. From this result it is clear that the SUSY algebra (6) is satisfied only on-shell. In a moment we will explain how it is possible to derive these additional terms using BV language. In the section 4 we will show that similar terms arise after integrating out auxiliary fields in the action (1).

Let us add BV antifields and ghosts to the classical action for SUSY QM

$$
\begin{array}{r}
S^{B V}=\int \frac{1}{2}\left(\partial_{t} x\right)^{2}+\frac{1}{2} D^{2}-i \bar{\psi} \partial_{t} \psi-\left(W^{\prime} D+W^{\prime \prime} \psi \bar{\psi}\right)+ \\
+(\varepsilon \bar{\psi}+\bar{\varepsilon} \psi) x^{*}-\varepsilon\left(i \partial_{t} x+D\right) \psi^{*}+\bar{\varepsilon}\left(-i \partial_{t} x+D\right) \bar{\psi}^{*}+\left(i \bar{\varepsilon} \partial_{t} \psi-i \varepsilon \partial_{t} \bar{\psi}\right) D^{*}+ \\
+\eta \partial_{t} x x^{*}+\eta \partial_{t} \psi \psi^{*}+\eta \partial_{t} \bar{\psi} \bar{\psi}^{*}+\eta \partial_{t} D D^{*}+2 i \varepsilon \bar{\varepsilon} \eta^{*}
\end{array}
$$

The first line of this expression is the classical action for SUSY QM, the second one is BV structure of SUSY transformation (see (8) for the transformations of the component fields), the last line contains BV structure for translations in time (this is necessary to close the algebra of symmetry) and the term with the structure constants for the symmetry algebra (the last term). We would like to emphasize the difference between parameter $\epsilon^{\alpha}$ of SUSY transformation (see for example (10) ) and the ghost $\varepsilon_{\alpha}$ for SUSY transformation used in (11). The first one is odd variable, the second one is even. There is complete analogy with gauge theories here: parameter of gauge transformation is even, while parameter of BRST transformation (Faddeev-Popov ghost) is odd. The parity of the ghost for the transformation is always opposite to the parity of the parameter. The ghost field for translations is denoted by $\eta$ (odd variable), the BV anti-ghost for translations is $\eta^{*}$ (even variable).

Action (11) satisfies classical BV equation:

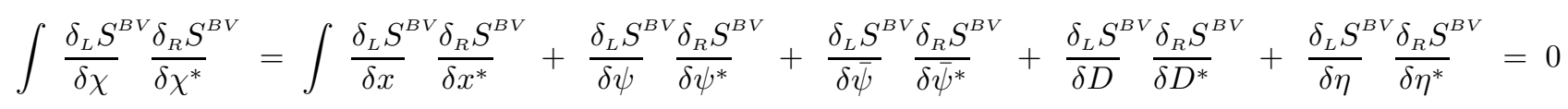

Here $\chi$ stands for all the fields. There are no terms arising from the variation over $\varepsilon$ and $\bar{\varepsilon}$ because the action $S^{B V}$ is independent of the antifields $\varepsilon^{*}$ and $\bar{\varepsilon}^{*}$. Integrating $\mathrm{BV}$ action over a lagrangian submanifold results into effective action which again satisfies BV equation (the simplistic explanation of this fact is given in the section 2 of [2], for the rigorous proof see [4]). Let us integrate over the auxiliary field $D$ on the lagrangian submanifold $D^{*}=0$. The result for the effective action is:

$$
\begin{array}{r}
S^{\text {eff }}=\int \frac{1}{2}\left(\partial_{t} x\right)^{2}-i \bar{\psi} \partial_{t} \psi-\frac{1}{2} W^{\prime 2}-W^{\prime \prime} \psi \bar{\psi}+ \\
+(\varepsilon \bar{\psi}+\bar{\varepsilon} \psi) x^{*}-i \varepsilon \partial_{t} x \psi^{*}-i \bar{\varepsilon} \partial_{t} x \bar{\psi}^{*}-W^{\prime}\left(\varepsilon \psi^{*}-\bar{\varepsilon} \bar{\psi}^{*}\right)+ \\
+\eta \partial_{t} x x^{*}+\eta \partial_{t} \psi \psi^{*}+\eta \partial_{t} \bar{\psi} \bar{\psi}^{*}+2 i \varepsilon \bar{\varepsilon} \eta^{*}- \\
-\frac{1}{2}\left(\varepsilon \psi^{*}-\bar{\varepsilon} \bar{\psi}^{*}\right)^{2}
\end{array}
$$

Similarly to (11) in the first line we have classical action, in the second one - SUSY transformations of the remaining fields, the third line contains BV structure for translations and the structure constants term.

The most interesting term appears in the last line. It is quadratic in the antifields and quadratic in the ghosts $\varepsilon$ and $\bar{\varepsilon}$. Let us decompose the effective action $S^{\text {eff }}$ into two parts $S^{\text {eff }}=S_{S}+S^{\text {add }}$, where $S^{a d d}=-\frac{1}{2}\left(\varepsilon \psi^{*}-\bar{\varepsilon} \bar{\psi}^{*}\right)^{2}$. Subscript " $S$ " in $S_{S}$ denotes the action with standard (linear in antifields) coupling to ghosts. Since (12) is obtained from the integration of BV action over the lagrangian submanifold, $S^{e f f}$ satisfies classical BV equation, which can be written as

$$
0=\int \frac{\delta_{L} S^{e f f}}{\delta \chi^{n}}{\frac{\delta_{R}}{\delta \chi_{n}^{*}}}^{\text {eff }}=\int \frac{\delta_{L} S_{S}}{\delta \chi^{n}} \frac{\delta_{R} S_{S}}{\delta \chi_{n}^{*}}+\frac{\delta_{L} S_{S}}{\delta \psi} \frac{\delta_{R} S^{\text {add }}}{\delta \psi^{*}}+\frac{\delta_{L} S_{S}}{\delta \bar{\psi}} \frac{\delta_{R} S^{\text {add }}}{\delta \bar{\psi}^{*}}
$$

Taking into account explicit expressions for $S^{a d d}$ and $S_{S}$ one can rewrite this result as

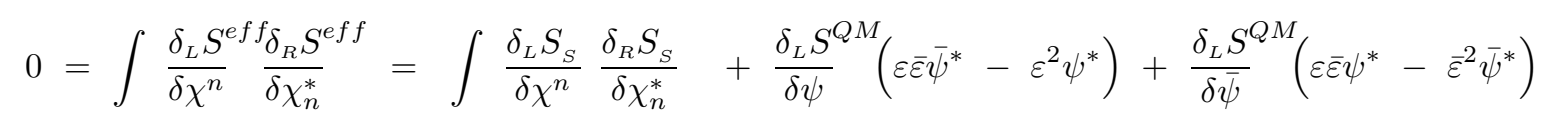

where $S^{Q M}$ is the classical action for quantum mechanics (7). One can see that the last two terms vanish on the equations of motion for the fields $\psi$ and $\bar{\psi}$. Reducing equation (14) to the solutions of the classical equations of motion one can obtain

$$
\left.\int \frac{\delta_{L} S_{S}}{\delta \chi^{n}} \frac{\delta_{R} S_{S}}{\delta \chi_{n}^{*}}\right|_{\text {on the e.o.m. }}=0
$$


which is the condition of SUSY invariance. This condition however is valid only on-shell.

What is important for us from this calculation is that appearance of $\varepsilon^{2}\left(\chi^{*}\right)^{2}$ terms in the effective action signals the descent of off-shell invariance of the fundamental action down to on-shell invariance of the effective action.

\subsection{General case}

Though this effect was illustrated using the simplest possible example - supersymmetric QM interpretation of these terms (quadratic in antifields and in the ghosts for SUSY) is universal and does not depend on the particular theory. To demonstrate this one can write the general structure of effective action as

$$
S^{e f f}=S^{c l}+c^{\alpha} V_{\alpha}^{n} \chi_{n}^{*}-\frac{1}{2} f_{\alpha \beta}^{\gamma} c^{\alpha} c^{\beta} c_{\gamma}^{*}+S^{a d d},
$$

where we used the notation $V_{\alpha}^{n}=Q_{\alpha} \chi^{n}$ in the term describing the transformation of the component fields. The algebra of symmetry is given by $\left[Q_{\alpha}, Q_{\beta}\right]=f_{\alpha \beta}^{\gamma} Q_{\gamma}$ and $c^{\alpha}$ are ghosts for this algebra. One can straightforwardly plug this action into the classical BV equation and collect the terms linear in antifields. The result is given by

$$
c^{\alpha} V_{\alpha}^{k} c^{\beta} \frac{\delta V_{\beta}^{n}}{\delta \chi^{k}} \chi_{n}^{*}-\frac{1}{2} V_{\alpha}^{n} \chi_{n}^{*} f_{\beta \gamma}^{\alpha} c^{\beta} c^{\gamma}+\frac{\delta S^{c l}}{\delta \chi^{k}} \frac{\delta S^{a d d}}{\delta \chi_{k}^{*}}=0 .
$$

Varying this expression w.r.t. $c^{\alpha}, c^{\beta}$ and $\chi_{n}^{*}$ one can find

$$
V_{\alpha}^{k} \frac{\delta V_{\beta}^{n}}{\delta \chi^{k}}+V_{\beta}^{k} \frac{\delta V_{\alpha}^{n}}{\delta \chi^{k}}-f_{\alpha \beta}^{\gamma} V_{\gamma}^{n}+\frac{\delta S^{c l}}{\delta \chi^{k}} \frac{\delta^{(4)} S^{a d d}}{\delta c^{\alpha} \delta c^{\beta} \delta \chi_{k}^{*} \delta \chi_{n}^{*}}=0
$$

which is equivalent to

$$
\left[Q_{\alpha}, Q_{\beta}\right] \chi^{n}=f_{\alpha \beta}^{\gamma} Q_{\gamma} \chi^{n}-\frac{\delta S^{c l}}{\delta \chi^{k}} \frac{\delta^{(4)} S^{a d d}}{\delta c^{\alpha} \delta c^{\beta} \delta \chi_{k}^{*} \delta \chi_{n}^{*}}=0
$$

and coincides with (10): the commutator of $\delta_{1}$ and $\delta_{2}$ is connected with the commutator of $Q$.

Thus the non-standard terms (quadratic in antifields) in BV action are in one-to-one correspondence with the corrections proportional to the equations of motion (10). This result is not new. For the non-complete list of references on the subject see [7].

\section{Wess-Zumino Gauge}

In this section we realize the gauge fixing procedure using the BV language in the simple and well known example: Wess-Zumino gauge in $\mathcal{N}=1$ four dimensional super-Maxwell theory. We find non-standard terms in the solution of BV Master Equation responsible for the fact that the Wess-Zumino gauge is not supersymmetric (the SUSY transformation of the vector multiplet in the Wess-Zumino gauge gives the fields which are absent in this gauge; to restore the Wess-Zumino gauge one should make an appropriate gauge transformation). In the next section we will demonstrate that exactly these terms appear in the effective action for (1) after evaluation on the cohomologies of $Q$ operator. This observation will lead to the conclusion that the action (1) contains the full multiplet of auxiliary fields needed to restore the SUSY invariance, while in the effective action these fields are integrated out.

\subsection{Gauge-fixing procedure in BV description}

In this subsection we will show that restriction of the Master Action of BV formalism to the certain lagrangian submanifold gives Faddeev-Popov action in the fixed gauge. As an example consider the BV action for the gauge invariant action $S^{c l}$ which depends only on the gauge field (no matter fields):

$$
S^{B V}=S^{c l}+\int D_{\mu}^{a c} c^{c}\left(A_{\mu}^{a}\right)^{*}-\frac{1}{2} f_{b c}^{a} c^{b} c^{c}\left(c_{a}\right)^{*}
$$

We are going to restrict this action to the certain lagrangian submanifold $\mathcal{L}_{f}$. The definition of $\mathcal{L}_{f}$ is given by:

$$
\left\{\begin{array}{l}
\left(c^{a}\right)^{*}=0 \\
f^{a}(A)=0 \\
\left(A_{\mu}^{a}\right)^{*}=-\frac{\partial f^{b}}{\partial A_{\mu}^{a}} \bar{c}^{b} \\
c^{a}-\text { is not restricted }
\end{array}\right.
$$


Thus coordinates on this submanifold are $c^{a}, \bar{c}^{a}$ and $A_{\mu}^{a}$ restricted by the constraint $f^{a}(A)=0$. The first coordinate $c^{a}$ is not restricted, while there is the constraint $f^{a}(A)=0$, imposed on the field $A_{\mu}^{a}$. The additional degree of freedom appearing in the field $\left(A_{\mu}^{a}\right)^{*}$ is parameterized by $\bar{c}^{b}$. As we will show in a moment this coordinate $\bar{c}^{b}$ on the submanifold is nothing but Faddeev-Popov antighost field. It is straightforward to check that $(20)$ is indeed a lagrangian submanifold:

$$
\begin{gathered}
\delta \chi_{n}^{*} \wedge \delta \chi^{n}=\delta c^{*} \wedge \delta c+\delta\left(A_{\mu}^{a}\right)^{*} \wedge \delta A_{\mu}^{a}=\delta A_{\mu}^{a} \wedge \delta\left(\frac{-\partial f^{b}}{\partial A_{\mu}^{a}} \bar{c}^{b}\right)= \\
=-\delta A_{\mu}^{a} \wedge \frac{\partial^{2} f^{b}}{\partial A_{\nu}^{c} \partial A_{\mu}^{a}} \bar{c}^{b} \delta A_{\nu}^{c}-\delta A_{\mu}^{a} \wedge \delta \bar{c}^{b} \frac{\partial f^{b}}{\partial A_{\mu}^{a}}=\frac{\partial^{2} f^{b}}{\partial A_{\nu}^{c} \partial A_{\mu}^{a}} \bar{c}^{b} \delta A_{\mu}^{a} \wedge \delta A_{\nu}^{c}-\delta f_{b} \wedge \delta \bar{c}^{b}=0
\end{gathered}
$$

We start with BV symplectic form written for all the fields and antifields of the theory. In the second equality we used $\left(c^{a}\right)^{*}=0$ and plugged $\left(A_{\mu}^{a}\right)^{*}$ from $(20)$. In the next equality we apply operator $\delta$ to $\frac{\partial f_{b}}{\partial A_{\mu}^{a}}$ and to $\bar{c}^{b}$. The first term in the last equality vanishes because $\frac{\partial^{2} f_{b}}{\partial A_{\nu}^{c} \partial A_{\mu}^{a}}$ is symmetric under interchange $A_{\mu}^{a} \leftrightarrow A_{\nu}^{c}$ while $\delta A_{\mu}^{a} \wedge \delta A_{\nu}^{c}$ is antisymmetric. The second term is equal to zero because of the constraint $f^{b}=0$ in (20).

Restricting the action (19) to the lagrangian submanifold (20) one can obtain

$$
\left.S^{B V}\right|_{\mathcal{L}_{f}}=S^{c l}+\int D_{\mu}^{a c} c^{c}\left(A_{\mu}^{a}\right)^{*}-\left.\frac{1}{2} g f_{b c}^{a} c^{b} c^{c}\left(c^{a}\right)^{*}\right|_{\mathcal{L}_{f}}=S^{c l}-\left.D_{\mu}^{a c} c^{c} \frac{\partial f_{b}}{\partial A_{\mu}^{a}} \bar{c}^{b}\right|_{f_{b}(A)=0}
$$

which is Faddeev-Popov action in the fixed gauge and the coordinate on the lagrangian submanifold $\bar{c}^{b}$ is Faddeev-Popov antighost field.

Summarizing this calculation one can see that to fix the certain gauge one should plug the gauge restriction on the fields into the action, integrate over the corresponding antifield, introducing the coordinate on the lagrangian submanifold according to (20), and put the BV antifield for the ghost equal to zero, integrating over the ghost.

\subsection{Gauge-fixing of the Wess-Zumino gauge}

We start from a set of definitions for the SUSY multiplets. We use the standard 2-d notations for the superfields (see for example [9]). In these notations the chiral multiplet is given by

$$
\begin{gathered}
\Lambda=(a+i b)(y)+\theta \psi(y)-\theta \theta F(y)= \\
=(a+i b)+\theta \psi+i \theta \sigma^{\mu} \bar{\theta} \partial_{\mu}(a+i b)-\theta \theta F-\frac{i}{2} \theta \theta \partial_{\mu} \psi \sigma^{\mu} \bar{\theta}-\frac{1}{4} \theta \theta \overline{\theta \theta} \partial^{2}(a+i b)
\end{gathered}
$$

the contraction of indices is given by $\theta \theta=\theta^{\alpha} \theta_{\alpha}$, while $\overline{\theta \theta}=\bar{\theta}_{\dot{\alpha}} \bar{\theta}^{\dot{\alpha}}$ and $y^{\mu}=x^{\mu}+i \theta \sigma^{\mu} \bar{\theta}$. The SUSY variation of component fields can be found by direct application of SUSY charges:

$$
\begin{gathered}
Q_{\alpha}=\frac{\partial}{\partial \theta^{\alpha}}-i \sigma_{\alpha \dot{\beta}}^{\mu} \bar{\theta}^{\dot{\beta}} \partial_{\mu} \\
\bar{Q}_{\dot{\alpha}}=-\frac{\partial}{\partial \bar{\theta}^{\dot{\alpha}}}+i \theta^{\beta} \sigma_{\beta \dot{\alpha}}^{\mu} \partial_{\mu}
\end{gathered}
$$

(here $\partial_{\mu}$ denotes the derivative w.r.t. $x^{\mu}$ ) according to the rule

$$
\delta \Lambda=(\epsilon Q+\overline{\epsilon Q}) \Lambda
$$

Performing simple calculations one can find:

$$
\begin{array}{r}
\delta a=\frac{1}{2}(\epsilon \psi+\overline{\epsilon \psi}) \\
\delta b=\frac{i}{2}(\overline{\epsilon \psi}-\epsilon \psi) \\
\delta \psi=-2 \epsilon F-2 i \partial_{\mu}(a+i b) \bar{\epsilon} \bar{\sigma}^{\mu} \\
\delta F=-i \bar{\epsilon} \bar{\sigma}^{\mu} \partial_{\mu} \psi
\end{array}
$$

Similar computation for the vector multiplet

$$
\begin{array}{r}
V(x, \theta, \bar{\theta})=C+i \theta \chi-i \overline{\theta \chi}+\theta \sigma^{\mu} \bar{\theta} A_{\mu}+\frac{i}{2} M \theta \theta-\frac{i}{2} \overline{M \theta \theta}+ \\
+i \theta \theta\left(\overline{\theta \lambda}+\frac{i}{2} \bar{\theta} \bar{\sigma}^{\mu} \partial_{\mu} \chi\right)-i \overline{\theta \theta}\left(\theta \lambda+\frac{i}{2} \theta \sigma^{\mu} \partial_{\mu} \bar{\chi}\right)+\frac{1}{2} \theta \theta \overline{\theta \theta}\left(D-\frac{1}{2} \partial^{2} C\right)
\end{array}
$$


gives the following component transformations:

$$
\begin{array}{r}
\delta C=i \epsilon \chi-i \overline{\epsilon \chi} \\
\delta \chi=\epsilon M-\partial_{\mu} C \bar{\epsilon} \bar{\sigma}^{\mu}+i A_{\mu} \bar{\epsilon} \bar{\sigma}^{\mu} \\
\delta A_{\mu}=-\left(\epsilon \partial_{\mu} \chi\right)+i\left(\epsilon \sigma^{\mu} \bar{\lambda}\right)-\overline{\epsilon \partial_{\mu} \chi}+i \bar{\epsilon} \bar{\sigma}^{\mu} \lambda \\
\delta \lambda=i \epsilon D-\frac{1}{2} \epsilon\left(\sigma^{\nu} \bar{\sigma}^{\mu}-\sigma^{\mu} \bar{\sigma}^{\nu}\right) \partial_{\mu} A_{\nu} \\
\delta D=-\epsilon \sigma^{\mu} \partial_{\mu} \bar{\lambda}+\bar{\epsilon} \bar{\sigma}^{\mu} \partial_{\mu} \lambda \\
\delta M=2 \overline{\epsilon \lambda}+2 i\left(\bar{\epsilon} \bar{\sigma}^{\mu} \partial_{\mu} \chi\right)
\end{array}
$$

It is well known that the gauge transformation for the vector multiplet is given by:

$$
V \longrightarrow V+\frac{1}{2}(\Lambda+\bar{\Lambda})
$$

which in component fields gives:

$$
\begin{array}{r}
\delta C=a \\
\delta \chi=-\frac{i}{2} \psi \\
\delta \bar{\chi}=\frac{i}{2} \bar{\psi} \\
\delta A_{\mu}=-\partial_{\mu} b \\
\delta \bar{M}=-F \\
\delta M=-\bar{F}, \quad \begin{array}{l}
\delta \bar{\lambda}=\delta D=0
\end{array}
\end{array}
$$

Now we are going to demonstrate how it is possible to fix the Wess-Zumino gauge in the abelian super Maxwell theory using BV formalism. We will show that after the gauge fixing some non-standard terms appear in the BV action. These terms are responsible for the fact that the Wess-Zumino gauge is not supersymmetric commutator of two SUSY transformations, should be accompanied by the appropriate gauge transformation to return into the Wess-Zumino gauge. As before we introduce the ghosts for the SUSY transformations: $\varepsilon$ and $\bar{\varepsilon}$ and the ghosts for translations $\eta^{\mu}$. The full Master Action of BV formalism can be schematically written as:

$$
\begin{gathered}
S^{B V}=\int-\frac{1}{4} F_{\mu \nu}^{2}-i \lambda \sigma^{\mu} \partial_{\mu} \bar{\lambda}+\frac{1}{2} D^{2}+ \\
+\frac{1}{2}(\Lambda+\bar{\Lambda}) V^{*}+\left(\varepsilon Q+\overline{\varepsilon Q}+\eta^{\mu} \partial_{\mu}\right) V V^{*}+\left(\varepsilon Q+\overline{\varepsilon Q}+\eta^{\mu} \partial_{\mu}\right) \Lambda \Lambda^{*}-2 i \eta_{\mu}^{*}\left(\varepsilon \sigma^{\mu} \bar{\varepsilon}\right)
\end{gathered}
$$

In the first line of this expression the classical abelian gauge invariant action is written. The second line contains the gauge and SUSY transformation of all the fields as well as the structure constant term resulting from the anticommutator of the SUSY charges $\left(\left\{Q_{\alpha}, \bar{Q}_{\dot{\alpha}}\right\}=2 i \sigma_{\alpha \dot{\alpha}}^{\mu} \partial_{\mu}\right)$. To write explicit expressions in the component fields one should take the component transformations from $(23),(25),(26)$ and multiply them by the appropriate antifield. For example one of the terms resulting from $\frac{1}{2}(\Lambda+\bar{\Lambda}) V^{*}$ gives $-\partial_{\mu} b\left(A_{\mu}\right)^{*}$ (see the fourth line of (26)).

WARNING. The fields of chiral multiplet are ghosts for the gauge transformation. Hence their parities are opposite to the standard parities of the component fields in the chiral multiplet. The fields $a, b, F, \bar{F}$ are odd, while $\psi$ and $\bar{\psi}$ are even.

Now we are going to fix the Wess-Zumino gauge $C=0, \chi=0, M=0$ using the procedure discussed in the subsection 3.1. To do this one should put the fields: $C, \chi, \bar{\chi}, M, \bar{M}$ equal to zero as well as antighosts: $a^{*}$, $\psi^{*}, \bar{\psi}^{*}, F^{*}, \bar{F}^{*}$ and integrate over the antifields $C^{*}, \chi^{*}, \bar{\chi}^{*}, M^{*}, \bar{M}^{*}$ as well as over the ghosts $a, \psi, \bar{\psi}, F, \bar{F}$. Direct computation taking into account the parities of all the fields gives ${ }^{1}$

$$
\begin{array}{r}
S^{e f f}=\int\left[-\frac{1}{4} F_{\mu \nu}^{2}-i \lambda \sigma^{\mu} \partial_{\mu} \bar{\lambda}+\frac{1}{2} D^{2}-\partial_{\mu} b\left(A_{\mu}\right)^{*}+i\left(\varepsilon \sigma^{\mu} \bar{\lambda}+\lambda \sigma^{\mu} \bar{\varepsilon}\right) A_{\mu}^{*}-\left(\varepsilon \sigma^{\mu} \partial_{\mu} \bar{\lambda}-\partial_{\mu} \lambda \sigma^{\mu} \bar{\varepsilon}\right) D^{*}\right. \\
+\left(i \varepsilon D-\frac{1}{2} \varepsilon\left(\sigma^{\mu} \bar{\sigma}^{\nu}-\sigma^{\nu} \bar{\sigma}^{\mu}\right) \partial_{\mu} A_{\nu}\right) \lambda^{*}+\left(-i \bar{\varepsilon} D-\frac{1}{2}\left(\bar{\sigma}^{\nu} \sigma^{\mu}-\bar{\sigma}^{\mu} \sigma^{\nu}\right) \bar{\varepsilon} \partial_{\mu} A_{\nu}\right) \bar{\lambda}^{*}+ \\
+\left(\eta^{\mu} \partial_{\mu} A^{\nu} A_{\nu}^{*}+\eta^{\mu} \partial_{\mu} D D^{*}+\eta^{\mu} \partial_{\mu} \lambda \lambda^{*}+\eta^{\mu} \partial_{\mu} \bar{\lambda} \bar{\lambda}^{*}+\eta^{\mu} \partial_{\mu} b b^{*}\right)-2 i\left(\varepsilon \sigma^{\mu} \bar{\varepsilon}\right) \eta_{\mu}^{*}- \\
\left.-2 i\left(\varepsilon \sigma^{\mu} \bar{\varepsilon}\right) A_{\mu} b^{*}\right]
\end{array}
$$

\footnotetext{
${ }^{1}$ Here we denote the gauge ghost by $b$ to avoid confusion with the first component $C$ of the vector multiplet.
} 
The aim of this calculation was to demonstrate the appearance of the last term $2 i \varepsilon \sigma^{\mu} \bar{\varepsilon} A_{\mu} b^{*}$. Following the logic mentioned in the end of the previous section one can check that these terms are responsible for the fact that the gauge which is fixed is not consistent with the supersymmetry. The algebra of SUSY is closed only up to the gauge transformation with parameter $\left(\varepsilon \sigma^{\mu} \bar{\varepsilon}\right) A_{\mu}$. In the next two sections we will show, that the terms discussed in the sections 2 and 3 are exactly those which arise when one integrates out auxiliary fields in the action (1) to obtain effective action. This will be shown for the model found in [2] and for Berkovits' 10-dimensional SYM theory.

\section{Superfield Formulation of Gauge Model of [2]}

In [2] we introduced the model that is believed to be the simplest example in the class of physically interesting models. Despite of its own interest this model can be considered as a toy model which inherits almost all the phenomena related to the descent of supersymmetry in case of 10-dimensional SYM, which is the main subject of the present paper. In [2] it was demonstrated that the classical part of effective BV action calculated for the theory (1) in case the system of quadratic constraints $f^{\mu}(\lambda)$ is given by:

$$
\begin{aligned}
& f_{1}=\lambda_{1} \lambda_{2} \\
& f_{2}=\lambda_{2} \lambda_{3} \\
& f_{3}=\lambda_{3} \lambda_{4} \\
& f_{4}=\lambda_{1}^{2} \\
& f_{5}=\lambda_{4}^{2}
\end{aligned}
$$

can be written as:

$$
\begin{aligned}
& S^{c l}=\int d^{2} x \operatorname{Tr}\left(\Phi F_{+-}+D_{+} \phi_{1} D_{-} \phi_{1}+D_{-} \phi_{2} D_{+} \phi_{2}-\frac{g}{\sqrt{2}} \phi_{1}\left\{\psi_{+}, \psi_{-}\right\}+i \frac{g}{\sqrt{2}} \phi_{2}\left\{\psi_{+}, \psi_{-}\right\}+\beta_{+} D_{-} \gamma_{+}+\right. \\
&\left.+\beta_{-} D_{+} \gamma_{-}+\bar{\psi}_{-} D_{+} \psi_{-}+\bar{\psi}_{+} D_{-} \psi_{+}+\bar{\chi}_{-} D_{+} \chi_{-}+\bar{\chi}_{+} D_{-} \chi_{+}+2 g \bar{\chi}_{-}\left[\gamma_{-}, \psi_{+}\right]+2 g \bar{\chi}_{+}\left[\gamma_{+}, \psi_{-}\right]\right)
\end{aligned}
$$

The aim of this section is to explain that this action is invariant under the global supersymmetry transformation on-shell in the same sense that $\mathcal{N}=1$ Yang-Mills action in the Wess-Zumino gauge is invariant under the supersymmetry transformation (the action is invariant and the SUSY algebra is closed up to an appropriate gauge transformation). Another point is that action (1) is the off-shell version of the theory (30) without elimination of auxiliary fields like $C, \psi, M$ in the Wess-Zumino gauge.

\subsection{Initial BV action}

Firstly we notice that there is an odd supersymmetry generator, built using the quadrics $f^{\mu}(\lambda)$, which anticommutes with the Berkovits operator

$$
Q_{B}=Q+\Phi=\lambda_{\alpha} \frac{\partial}{\partial \theta_{\alpha}}+\theta_{\alpha} \frac{\partial f^{\mu}}{\partial \lambda_{\alpha}} \partial_{\mu}
$$

This SUSY generator is given by:

$$
Q_{\alpha}^{S U S Y}=\frac{\partial}{\partial \theta^{\alpha}}-\frac{1}{2} \theta_{\beta} \frac{\partial^{2} f^{\mu}}{\partial \lambda_{\beta} \partial \lambda_{\alpha}} \partial_{\mu}
$$

By straightforward calculation one can see that

$$
\left\{Q_{B}, Q_{\alpha}^{S U S Y}\right\}=\frac{\partial f^{\mu}}{\partial \lambda_{\alpha}} \partial_{\mu}-\frac{1}{2} \lambda_{\beta} \frac{\partial^{2} f^{\mu}}{\partial \lambda_{\beta} \partial \lambda_{\alpha}} \partial_{\mu}=0
$$

This is true, because the functions $f^{\mu}(\lambda)$ are quadratic in $\lambda_{\alpha}$. Substituting explicit expressions for quadrics $f^{\mu}(\lambda)$ one can find the following expressions for the supersymmetry generators(we omit the superscripts SUSY):

$$
\begin{array}{ll}
Q_{1}=\frac{\partial}{\partial \theta_{1}}-2 \theta_{1} \partial_{+} & Q_{2}=\frac{\partial}{\partial \theta_{2}} \\
Q_{4}=\frac{\partial}{\partial \theta_{4}}-2 \theta_{4} \partial_{-} & Q_{3}=\frac{\partial}{\partial \theta_{3}}
\end{array}
$$


We remind that following [2] we consider the reduction from 5-dimensional space to 2-dimensions, putting $\partial_{1}=\partial_{2}=\partial_{3}=0$ and $\partial_{4}=\partial_{+}, \partial_{5}=\partial_{-}$. We are going to concentrate our consideration on the first non-trivial generator $Q_{1}$ which forms the closed algebra with the generator $\partial_{+}$. The commutation relations are:

$$
\begin{gathered}
\left\{Q_{1}, Q_{1}\right\}=-4 \partial_{+} \\
{\left[Q_{1}, \partial_{+}\right]=0} \\
{\left[\partial_{+}, \partial_{+}\right]=0}
\end{gathered}
$$

The idea is to add the sources $\varepsilon$ and $\eta$ for the generators $Q_{1}$ and $\partial_{+}$to the fundamental action (1) to form the BV action:

$$
S^{S U S Y}=\int \operatorname{Tr}\left(<\mathcal{P}, Q_{B} \mathcal{A}>+g<\mathcal{P}, \mathcal{A}^{2}>+\varepsilon<\mathcal{P}, Q_{1} \mathcal{A}>+\eta<\mathcal{P}, \partial_{+} \mathcal{A}>-2 \varepsilon^{2} \eta^{*}\right)
$$

We remind that the field $\mathcal{A}$ is a generic superfield build out of $\lambda^{\alpha}, \theta^{\alpha}$ and component fields. $\mathcal{P}$ is a generic element of the space dual to the space of superfields (dual superfield). The component fields of $\mathcal{A}$ and $\mathcal{P}$ are different. Canonical pairing $\langle$,$\rangle is defined as \left\langle\underline{e}^{a}, e_{b}\right\rangle=\delta_{b}^{a}$. Here $e_{a}$ is a basis in the space of $\lambda$ and $\theta$ and $\underline{e}^{a}$ is dual basis in the dual space.

By direct substitution one can check that this action satisfies classical BV equation

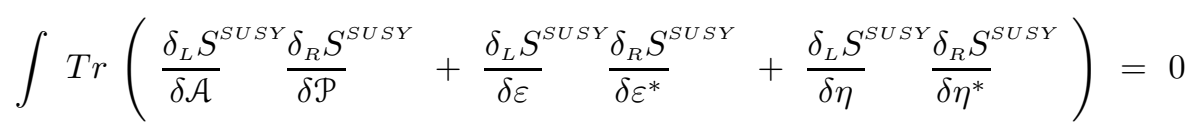

The action does not depend on $\varepsilon^{*}$, hence the second term in the Master Equation is automatically zero. Action (34) satisfies this equation under the following conditions:

1. Operator $Q_{B}$ is nilpotent $Q_{B}^{2}=0$.

2. Generators $Q_{1}$ and $\partial_{+}$satisfy the commutation relations (33).

3. Operator $Q_{B}$ commutes with the generators as: $\left\{Q_{B}, Q_{1}\right\}=0$ and $\left[Q_{B}, \partial_{+}\right]=0$.

4. Operators $Q_{B}, Q_{1}$ and $\partial_{+}$differentiate multiplication of superfields $\mathcal{A}$, i.e. satisfy Leibnitz identity.

5. The field $\varepsilon$ is even, the field $\eta$ is odd, $\mathcal{A}$ and $\mathcal{P}$ are odd and even superfields respectively.

6. The fields $\varepsilon$ and $\eta$ (as well as $\varepsilon^{*}$ and $\eta^{*}$ ) are ghosts for the global symmetry, hence do not depend on space-time coordinates.

We are going to integrate out all the fields in the action (34) from the complement to the space of cohomologies $\mathcal{H}(Q)$. Here operator $Q=\lambda_{\alpha} \frac{\partial}{\partial \theta_{\alpha}}$ is the first term in (31). These cohomologies were calculated in the paper [2] using the tower of fundamental relations, see also [10] for the same calculation via localization technique. Thus we are going to decompose the fields

$$
\begin{aligned}
\mathcal{A} & =\mathrm{A}+a \\
\mathcal{P} & =\mathrm{P}+p
\end{aligned}
$$

onto the superfield A of cohomologies of $\mathcal{H}(Q)$ and the superfield $a$ on the complement to $\mathcal{H}(Q)$. The same decomposition on $\mathrm{P}$ and $p$ is done in the dual space. The idea is to integrate over $a$ and $p$ to obtain effective action on the component fields of $\mathrm{A}$ and $\mathrm{P}$.

The sources $\varepsilon$ and $\eta$ for the supersymmetry and translations will allow to control the supersymmetric properties of the effective action. The next subsection contains the calculation of effective action using the Feynman diagram technique established in [2]. 


\subsection{Calculation of Effective action}

\begin{tabular}{|c|c|c|}
\hline Polarization & Field & Antifield \\
\hline 1 & $c$ & $\widetilde{c}$ \\
& & \\
\hline$\lambda_{1} \theta_{2}+\lambda_{2} \theta_{1}$ & $\gamma_{+}$ & $\widetilde{\gamma}_{+}$ \\
$\lambda_{2} \theta_{3}+\lambda_{3} \theta_{2}$ & $\varphi$ & $\widetilde{\varphi}$ \\
$\lambda_{3} \theta_{4}+\lambda_{4} \theta_{3}$ & $\gamma_{-}$ & $\widetilde{\gamma}_{-}$ \\
$\lambda_{1} \theta_{1}$ & $A_{+}$ & $\widetilde{A}_{+}$ \\
$\lambda_{4} \theta_{4}$ & $A_{-}$ & $\widetilde{A}_{-}$ \\
& & \\
\hline & & \\
$\lambda_{1} \theta_{1} \theta_{2}$ & $\psi_{+}$ & $\widetilde{\psi}_{+}$ \\
$\lambda_{4} \theta_{4} \theta_{3}$ & $\psi_{-}$ & $\widetilde{\psi}_{-}$ \\
$\lambda_{2} \theta_{3} \theta_{1}$ & $\chi_{+}$ & $\widetilde{\chi}_{+}$ \\
$\lambda_{3} \theta_{2} \theta_{4}$ & $\chi_{-}$ & $\widetilde{\chi}_{-}$ \\
\hline & & \\
\hline$\lambda_{1} \lambda_{4} \theta_{1} \theta_{4}$ & $\varphi_{1}$ & $\widetilde{\varphi}_{1}$ \\
$\lambda_{1} \lambda_{4} \theta_{4} \theta_{2}+\lambda_{2} \lambda_{4} \theta_{4} \theta_{1}$ & $\varphi_{2}$ & $\widetilde{\varphi}_{2}$ \\
$\lambda_{1} \lambda_{3} \theta_{1} \theta_{4}+\lambda_{1} \lambda_{4} \theta_{1} \theta_{3}$ & $\varphi_{3}$ & $\widetilde{\varphi}_{3}$ \\
\hline$\lambda_{1} \lambda_{4} \theta_{1} \theta_{4} \theta_{3}$ & & \\
$\lambda_{1} \lambda_{4} \theta_{1} \theta_{2} \theta_{4}$ & $\varphi_{4}$ & $\widetilde{\varphi}_{4}$ \\
$\lambda_{1} \lambda_{3} \theta_{1} \theta_{2} \theta_{4}+\lambda_{1} \theta_{2} \theta_{3} \theta_{4} \theta_{1} \theta_{2} \theta_{3}$ & $\varphi_{6}$ & $\widetilde{\varphi}_{5}$ \\
$\lambda_{2} \lambda_{4} \theta_{1} \theta_{4} \theta_{3}+\lambda_{1} \lambda_{4} \theta_{2} \theta_{4} \theta_{3}$ & $\varphi_{7}$ & $\widetilde{\varphi}_{7}$ \\
\hline & $\varphi_{8}$ & $\widetilde{\varphi}_{8}$ \\
\hline
\end{tabular}

For the calculations in this section we use the notations of section 6 of [2]. The physical degrees of freedom - representatives of cohomologies of operator $Q$, are presented in the table. The first column gives polarizations for the component fields, the second one - notations for the component fields of $A$, the last one - notations for the component fields of $\mathrm{P}$. To compute effective action one has to sum up all connected tree diagrams with the external legs being the component fields of A (input lines in the diagram) and $P$ (the output line). As it was explained in [2] each diagram can have only one output line.

Conducting this calculation one has to remember that the diagrams having the propagator (wavy line), like the diagrams $4,5,6,7$ in the figure 2 , should be added with the relative $m i$ nus sign to the diagrams without propagator, like the diagrams $1,2,3$ in the figure 2. The simplest argument for this can be given in Minkowski space. Each operator insertion, like $\Phi, \varepsilon Q_{1}$ or $\eta \partial_{+}$, contributes a factor of $i$ (complex unity), coming from the exponent, the propagator (wavy line) also contributes a factor of $i$. Hence the diagrams 1,2,3 are proportional to $i$, while the diagrams $4,5,6,7$ are proportional to $-i$ (there are two operators and one propagator). That is why to find the result for the effective action one has to sum all the diagrams without propagator and subtract all the diagrams with one propagator. It is straightforward to demonstrate that it is impossible to draw the diagrams with more than one propagator by calculating the degree in $\lambda$ and $\theta$ in the final expression before taking the projection onto cohomologies.

\subsubsection{Linear level}

Firstly, consider the linear problem (gauge coupling constant $g=0$ ). The list of the diagrams giving nonzero result is presented in the fig. 2.

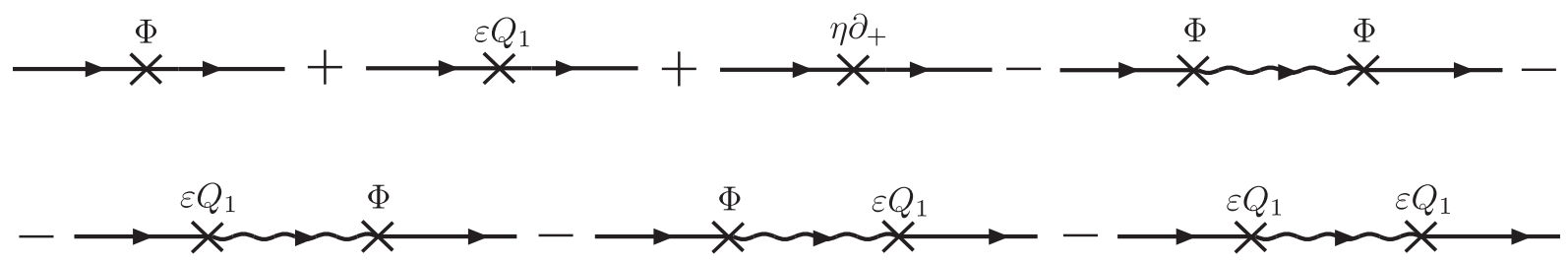

Figure 2: Linear level.

A lot of diagrams are absent in this figure. To prove this fact one has to count the degrees of lambda and theta. For example, the diagrams with three insertions of operator $\Phi$ are absent. This is true because each insertion of operator $\Phi$ gives multiplication by $\theta \lambda$, and these diagrams should have at least 2 propagators, each carrying degree $\theta / \lambda$. Thus these diagrams should change the degree in $\lambda$ and $\theta$ by: $\left(\frac{\theta}{\lambda}\right)^{2}(\lambda \theta)^{2}=\theta^{4}$. However, there are no two representatives of cohomologies, having equal number of $\lambda$ and difference 4 in the degree of $\theta$.

Another example of vanishing sub-diagrams is presented in the fig. 3. In the first fragment propagator is applied to cohomology, this diagram is equal to zero. Really, propagator gives non-zero result only if it is applied to $Q$-exact expression (see section 3 of [2] for details). The same is true for the second fragment because the image of the propagator has zero projection onto cohomologies. In the last fragment we meet square of propagator which is equal to zero. Returning to the diagrams in the fig. 2 we recall that the first and the last diagrams in the first line does not contain ghosts for SUSY or translations, hence they have been already calculated in [2]. Below we give a schematic illustration of the procedure for calculation of other diagrams. 


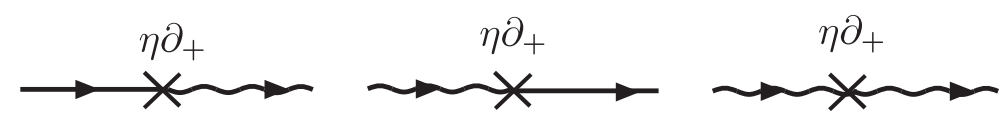

Figure 3: Forbidden subdiagrams.

1. The simplest one is with the insertion of $\eta \partial_{+}$operator(the third diagram in the fig. 2). Each of 18 fields contributes to this diagram in a trivial way: if input line is the certain field then output line exactly projected to its antifield.

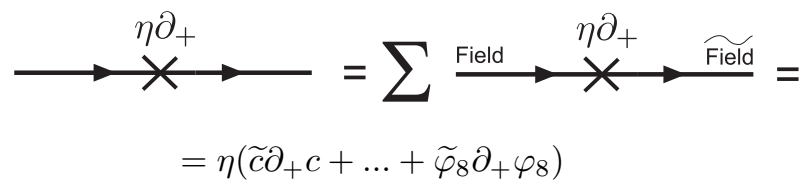

2. Consider the diagrams with the insertion of operator $Q_{1}$ (the second diagram in the fig. 2). Among all 18 fields of the theory only 8 give contribution to this diagram. They are: $\gamma_{+}, \chi_{+}, \psi_{+}, \varphi, \varphi_{2}, \varphi_{5}, \varphi_{7}, \varphi_{8}$. Consider for instance $\gamma_{+}$as an input line:

$$
\gamma_{+}: \varepsilon Q_{1}\left(\lambda_{1} \theta_{2}+\lambda_{2} \theta_{1}\right) \gamma_{+}=\varepsilon\left(\lambda_{2} \gamma_{+}-2 \lambda_{1} \theta_{1} \theta_{2} \partial_{+} \gamma_{+}\right) \stackrel{\text { Projection }}{\longrightarrow}-2 \varepsilon \widetilde{\psi}_{+} \partial_{+} \gamma_{+} .
$$

To project the result written on the l.h.s of the arrow one should look at the $7^{\text {th }}$ line of the table with the polarizations (cohomologies). Conducting the same procedure, one can obtain the contributions of all 8 fields to the effective action. The result is given by:

$$
\Delta S_{L i n}^{\left(Q_{1}\right)}=\int \operatorname{Tr}\left(-2 \varepsilon \widetilde{\psi}_{+} \partial_{+} \gamma_{+}+2 \varepsilon \widetilde{\varphi}_{8} \partial_{+} \varphi_{7}+2 \varepsilon \widetilde{\varphi}_{5} \partial_{+} \varphi_{2}+\frac{1}{2} \varepsilon \widetilde{\gamma}_{+} \psi_{+}-\frac{1}{2} \varepsilon \widetilde{\varphi} \chi_{+}-\frac{1}{2} \varepsilon \widetilde{\varphi}_{2} \varphi_{5}-\frac{1}{2} \varepsilon \widetilde{\varphi}_{7} \varphi_{8}\right)
$$

A little bit more difficult is to calculate the diagrams with the propagator. The propagator $K$ is defined in the section 3 of [2]. Roughly speaking it acts as follows: $K$, acting on representatives of cohomologies gives zero; being applied to exact expressions propagator gives the pre-image $(K(Q \omega)=\omega)$ of operator $Q$; propagator, being applied to certain non-closed expressions gives zero (see [2] for details).

3. The diagrams containing $\Phi$ and $Q_{1}$ (the $5^{\text {th }}$ and $6^{\text {th }}$ diagrams in the fig. 2 ). In case $\Phi Q_{1}$-diagrams ( $Q_{1}$ acts first), input lines are $\gamma_{+}, \varphi_{2}, \varphi_{7}$. Consider for example $\gamma_{+}$:

$$
\Phi K \varepsilon Q_{1}\left(\lambda_{1} \theta_{2}+\lambda_{2} \theta_{1}\right) \gamma_{+}=\varepsilon \Phi K\left(\lambda_{2} \gamma_{+}-2 \lambda_{1} \theta_{1} \theta_{2} \partial_{+} \gamma_{+}\right)=\varepsilon \Phi K\left(Q\left(\theta_{2} \gamma_{+}\right)-2 \lambda_{1} \theta_{1} \theta_{2} \partial_{+} \gamma_{+}\right)
$$

the second term in the r.h.s. is proportional to the cohomology and propagator acts on it as zero.

$$
\varepsilon \Phi K\left(Q\left(\theta_{2} \gamma_{+}\right)-2 \lambda_{1} \theta_{1} \theta_{2} \partial_{+} \gamma_{+}\right)=\varepsilon \Phi \theta_{2} \gamma_{+}=2 \varepsilon \lambda_{1} \theta_{1} \theta_{2} \partial_{+} \gamma_{+}+2 \varepsilon \lambda_{4} \theta_{4} \theta_{2} \partial_{-} \gamma_{+}
$$

In this simple example one can see the key feature of this calculation. The first term in the r.h.s. has the polarization of $\widetilde{\psi}_{+}$(see line 7 in the table) hence one can project this term to $2 \varepsilon \widetilde{\psi}_{+} \partial_{+} \gamma_{+}$. The second term is nonclosed, but it was mentioned before each expression has a chance to sum up with similar term from another diagram to form a closed result. This closed result can have a non-trivial projection onto cohomologies. The result for the remaining two input lines and $\gamma_{+}$is the following

$$
\Delta S_{L i n}^{\left(\Phi Q_{1}\right)}=\int \operatorname{Tr}\left(2 \varepsilon \widetilde{\varphi}_{8} \partial_{+} \varphi_{7}-2 \varepsilon \widetilde{\psi}_{+} \partial_{+} \gamma_{+}+2 \varepsilon \widetilde{\varphi}_{5} \partial_{+} \varphi_{2}\right) .
$$

Finally, in case $Q_{1} \Phi$ ( $\Phi$ acts first) there are no proper input fields resulting in the closed expression.

4. Diagrams containing $Q_{1} Q_{1}$. These diagrams are the most interesting in the sense, that they give terms proportional to $\varepsilon^{2}$. From the section 2 we know how to interpret this terms. Possible input lines for this diagram are: $A_{+}, \varphi_{1}, \varphi_{3}, \varphi_{4}, \varphi_{6}$. The resulting contribution to the effective action is

$$
\Delta S_{L i n}^{\left(Q_{1} Q_{1}\right)}=\int \operatorname{Tr}\left(-\varepsilon^{2} \widetilde{c} A_{+}-\varepsilon^{2} \widetilde{A}_{-} \varphi_{1}-\varepsilon^{2} \widetilde{\gamma}_{-} \varphi_{3}-\varepsilon^{2} \widetilde{\psi}_{-} \varphi_{4}-2 \varepsilon^{2} \widetilde{\chi}_{-} \varphi_{6}\right) .
$$

5. After all one should examine all nonclosed results coming from all the diagrams. There are six such terms. Corresponding in-lines are $\gamma_{+}, \gamma_{-}, A_{+}, A_{-}, \varphi$. Among these six terms only the field $\varphi$ is summed up 
into a non-zero contribution into effective action. One nonclosed part comes from $Q_{1} \Phi$ diagram, another part comes from nonpropagating diagram with $Q_{1}$ inserted.

$$
\begin{gathered}
\varepsilon Q_{1} \varphi=-2 \varepsilon\left(\lambda_{2} \theta_{1} \theta_{3}+\lambda_{3} \theta_{1} \theta_{2}\right) \partial_{+} \varphi \\
\varepsilon Q_{1} K \Phi \varphi=2 \varepsilon \lambda_{1} \theta_{2} \theta_{3} \partial_{+} \varphi \\
\varepsilon\left(Q_{1}-Q_{1} K \Phi\right) \varphi=-2 \varepsilon\left(\lambda_{2} \theta_{1} \theta_{3}+\lambda_{1} \theta_{2} \theta_{3}+\lambda_{3} \theta_{1} \theta_{2}\right) \partial_{+} \varphi=2 \varepsilon\left(2 \lambda_{2} \theta_{3} \theta_{1}-Q\left(\theta_{1} \theta_{2} \theta_{3}\right)\right) \stackrel{\text { Proj. }}{\longrightarrow} 4 \varepsilon \widetilde{\chi}_{+} \partial_{+} \varphi
\end{gathered}
$$

since projection to cohomologies annihilates exact expressions.

Now we are ready to write down the whole linear effective action with the sources $\varepsilon$ and $\eta$.

$$
\begin{aligned}
S_{\text {lin }}^{e f f}= & \int \operatorname{Tr}\left(\widetilde{\varphi}_{1}\left(2\left(\partial_{+} A_{-}-\partial_{-} A_{+}\right)\right)+\widetilde{\varphi}_{2}\left(2 \partial_{-} \gamma_{+}-\frac{1}{2} \varepsilon \varphi_{5}\right)+\widetilde{\varphi}_{3}\left(2 \partial_{+} \gamma_{-}\right)+\widetilde{\varphi}_{4}\left(2 \partial_{+} \psi_{-}\right)+\right. \\
& +\widetilde{\varphi}_{5}\left(2 \partial_{-} \psi_{+}+4 \varepsilon \partial_{+} \varphi_{2}\right)+\widetilde{\varphi}_{6}\left(\partial_{+} \chi_{-}\right)+\widetilde{\varphi}_{7}\left(\partial_{-} \chi_{+}-\frac{1}{2} \varepsilon \varphi_{8}\right)+\widetilde{\varphi}_{8}\left(+8 \partial_{+} \partial_{-} \varphi+4 \varepsilon \partial_{+} \varphi_{7}\right)+ \\
& +\widetilde{\gamma}_{+}\left(\frac{1}{2} \varepsilon \psi_{+}\right)+\widetilde{\gamma}_{-}\left(-\varepsilon^{2} \varphi_{3}\right)+\widetilde{\varphi}\left(-\frac{1}{2} \varepsilon \chi_{+}\right)+\widetilde{A}_{+}\left(2 \partial_{+} c\right)+\widetilde{A}_{-}\left(-\varepsilon^{2} \varphi_{1}+2 \partial_{-} c\right)+ \\
& +\widetilde{\psi}_{+}\left(-4 \varepsilon \partial_{+} \gamma_{+}\right)+\widetilde{\psi}_{-}\left(-\varepsilon^{2} \varphi_{4}\right)+\widetilde{\chi}_{+}\left(4 \varepsilon \partial_{+} \varphi\right)+\widetilde{\chi}_{-}\left(-2 \varepsilon^{2} \varphi_{6}\right)+\widetilde{c}\left(-\varepsilon^{2} A_{+}\right)+ \\
& \left.+\eta\left(\tilde{c} \partial_{+} c+\ldots+\widetilde{\varphi}_{8} \partial_{+} \varphi_{8}\right)-2 \eta^{*} \varepsilon^{2}\right)
\end{aligned}
$$

\subsubsection{Nonlinear level}

Fortunately there is quite small number of additional diagrams arising after switching on the interaction. They are depicted in fig. 4. All calculations are completely analogous to linear case.

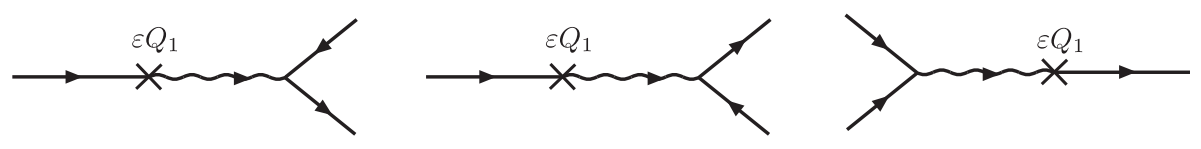

Figure 4: Nonlinear diagrams.

One can straightforwardly check that only «eg» order survives. As in the previous case two pairs of nonclosed constructions find each other and result into

$$
\Delta S_{\text {Nonlin }}^{1}=\int \operatorname{Tr}\left(2 \varepsilon g \widetilde{\chi}_{+}\left[A_{+}, \varphi\right]-4 \varepsilon g \widetilde{\chi}_{-}\left[\gamma_{+}, \gamma_{-}\right]\right)
$$

other terms are "purely" projected, i.e. each diagram gives closed result and can be projected separately without summing up with another diagram.

$$
\begin{aligned}
\Delta S_{\text {Nonlin }}^{2}=\int \operatorname{Tr} & \left(-2 \varepsilon g \widetilde{\psi}_{+}\left[A_{+}, \gamma_{+}\right]-2 \varepsilon g \widetilde{\varphi}_{5}\left[\varphi_{1}, \gamma_{+}\right]-2 \varepsilon g \widetilde{\varphi}_{6}\left[\varphi_{3}, \gamma_{+}\right]+2 \varepsilon g \widetilde{\varphi}_{8}\left[\varphi_{4}, \gamma_{+}\right]-\right. \\
& \left.-2 \varepsilon g \widetilde{\varphi}_{5}\left[\varphi_{2}, A_{+}\right]-2 \varepsilon g \widetilde{\varphi}_{8}\left[\varphi_{7}, A_{+}\right]+\frac{1}{2} \varepsilon g \widetilde{\varphi}_{8}\left\{\varphi_{1}, \chi_{+}\right\}-\frac{1}{2} \varepsilon g \widetilde{\varphi}_{7}\left[\varphi, \varphi_{1}\right]\right)
\end{aligned}
$$

Finally we collect together the results of the work [2] and additional terms with sources for SUSY. Effective lagrangian is given by

$$
\begin{aligned}
L^{e f f} & =\left[\Phi F_{+-}+\varphi_{8}\left\{D_{+}, D_{-}\right\} \varphi-g \varphi_{8}\left\{\psi_{+}, \psi_{-}\right\}+2 g \bar{\chi}_{-}\left[\gamma_{-}, \psi_{+}\right]+2 g \bar{\chi}_{+}\left[\gamma_{+}, \psi_{-}\right]+\beta_{+} D_{-} \gamma_{+}+\beta_{-} D_{+} \gamma_{-}\right. \\
& \left.+\bar{\psi}_{-} D_{+} \psi_{-}+\bar{\psi}_{+} D_{-} \psi_{+}+\bar{\chi}_{-} D_{+} \chi_{-}+\bar{\chi}_{+} D_{-} \chi_{+}\right]+\left[2 A_{+}^{*} \partial_{+} c+2 A_{-}^{*} \partial_{-} c+g\left(c^{*} c c+\ldots+\varphi_{8}^{*}\left\{\varphi_{8}, c\right\}\right)\right] \\
& +\eta\left[c^{*} \partial_{+} c+\ldots+\varphi_{8}^{*} \partial_{+} \varphi_{8}\right]+\varepsilon\left[2 \chi_{+}^{*} D_{+} \varphi-\varphi_{8} D_{+} \bar{\chi}_{+}^{*}-2 \psi_{+}^{*} D_{+} \gamma_{+}-2 \bar{\psi}_{-} D_{+} \beta_{+}^{*}+\frac{1}{2} \beta_{+} \bar{\psi}_{+}^{*}\right. \\
& +\chi_{+} \varphi_{8}^{*}+\frac{1}{2} \gamma_{+}^{*} \psi_{+}-\frac{1}{2} \varphi^{*} \chi_{+}-g\left(\bar{\chi}_{+}\left[\varphi, \Phi^{*}\right]-2 \bar{\psi}_{+}\left[\Phi^{*}, \gamma_{+}\right]-4 \bar{\chi}_{-}\left[\beta_{-}^{*}, \gamma_{+}\right]+\frac{1}{2} \varphi_{8}\left\{\Phi^{*}, \chi_{+}\right\}-2 \varphi_{8}\left[\gamma_{+}, \bar{\psi}_{-}^{*}\right]\right. \\
& \left.\left.+4 \psi_{+}^{*}\left[\gamma_{+}, \gamma_{-}\right]\right)\right]-2 \eta^{*} \varepsilon^{2}+\varepsilon^{2}\left[\gamma_{-}^{*} \beta_{-}^{*}+A_{-}^{*} \Phi^{*}+\psi_{-}^{*} \bar{\psi}_{-}^{*}+\chi_{-}^{*} \bar{\chi}_{-}^{*}-c^{*} A_{+}\right] .
\end{aligned}
$$


Here we turn to the physical notations as it was in [2]. Namely,

$$
\begin{gathered}
D_{+}=2 \partial_{+}+g\left[A_{+}, \cdot\right], \quad D_{-}=2 \partial_{-}+g\left[A_{-} \cdot \cdot\right], \\
F_{+-}=2\left(\partial_{-} A_{+}-\partial_{+} A_{-}\right)+g\left[A_{+}, A_{-}\right]
\end{gathered}
$$

and fields

$$
\begin{gathered}
c, A_{ \pm}, \ldots, \chi_{ \pm} \rightarrow c, A_{ \pm}, \ldots, \chi_{ \pm} \\
\widetilde{c}, \widetilde{A}_{ \pm}, \ldots, \widetilde{\chi}_{ \pm} \rightarrow c^{*}, A_{ \pm}^{*}, \ldots, \chi_{ \pm}^{*} \\
\widetilde{\varphi}_{1}, \widetilde{\varphi}_{2}, \ldots, \widetilde{\varphi}_{7}, \widetilde{\varphi}_{8} \rightarrow \Phi, \beta_{+}, \beta_{-}, \bar{\psi}_{-}, \bar{\psi}_{+}, 2 \bar{\chi}_{-}, 2 \bar{\chi}_{+}, \varphi_{8} \\
\varphi_{1}, \varphi_{2}, \ldots, \varphi_{7}, \varphi_{8} \rightarrow-\Phi^{*},-\beta_{+}^{*},-\beta_{-}^{*},-\bar{\psi}_{-}^{*},-\bar{\psi}_{+}^{*},-\frac{1}{2} \bar{\chi}_{-}^{*},-\frac{1}{2} \bar{\chi}_{+}^{*},-\varphi_{8}^{*}
\end{gathered}
$$

One can see that the terms discussed in sections 2 and 3 appear in the last brackets. According to the discussions above, these terms are responsible for the descent of the off-shell SUSY invariance of (1) down to on-shell SUSY invariance of (42).

\section{Berkovits' 10-d Super Yang-Mills}

In this section we apply the ideas developed earlier in this paper to the 10-d Super Yang-Mills theory [3], [15]. This theory is more interesting from the physical point of view than the model considered in the previous section. However, the off-shell description ${ }^{2}$ of this model is more complicated because of the necessity to make $Z_{2}$ projection on the space of fields.

According to the ideology from the previous sections one should calculate effective action for the theory

$$
S^{S U S Y}=\int \operatorname{Tr}\left(<\mathcal{P}, Q_{B} \mathcal{A}>+g<\mathcal{P}, \mathcal{A}^{2}>+<\mathcal{P}, \varepsilon^{\alpha} Q_{\alpha}^{s} \mathcal{A}>+<\mathcal{P}, \eta^{\mu} P_{\mu}^{s} \mathcal{A}>-\eta_{\mu}^{*}\left(\varepsilon \gamma^{\mu} \varepsilon\right)\right)
$$

\begin{tabular}{|c|c|c|c|}
\hline Polarization & $A$ & Dual polarization & $\mathrm{P}$ \\
\hline 1 & $c$ & $\underline{1}$ & $\widetilde{c}$ \\
\hline$\left(\lambda \gamma^{\mu} \theta\right)$ & $A_{\mu}$ & $\left(\lambda \bar{\gamma}^{\mu} \theta\right)$ & $\widetilde{A}_{\mu}$ \\
\hline$\left(\lambda \gamma^{\mu} \theta\right)\left(\theta \gamma^{\mu}\right)_{\alpha}$ & $\psi^{\alpha}$ & $\left(\lambda \gamma^{\mu} \theta\right)\left(\theta \gamma^{\mu}\right)_{\alpha}$ & $\widetilde{\psi}^{\alpha}$ \\
\hline$-16\left(\lambda \gamma^{\mu} \theta\right)\left(\lambda \gamma^{\nu} \theta\right)\left(\theta \gamma^{\mu \nu}\right)^{\alpha}$ & $\psi_{\alpha}^{*}$ & $-16\left(\overline{\left.\lambda \gamma^{\mu} \theta\right)\left(\lambda \gamma^{\nu} \theta\right)(\theta} \gamma^{\mu \nu}\right)^{\alpha}$ & $\widetilde{\psi}_{\alpha}^{*}$ \\
\hline $10\left(\lambda \gamma^{\mu} \theta\right)\left(\lambda \gamma^{\nu} \theta\right)\left(\theta \gamma_{\mu \nu \rho} \theta\right)$ & $A_{\rho}^{*}$ & $10\left(\overline{\left.\lambda \gamma^{\mu} \theta\right)\left(\lambda \gamma^{\nu} \theta\right)\left(\theta \gamma_{\mu \nu \rho} \theta\right)}\right.$ & $\widetilde{A}_{\rho}^{*}$ \\
\hline$\left(\lambda \gamma^{\mu} \theta\right)\left(\lambda \gamma^{\nu} \theta\right)\left(\lambda \gamma^{\rho} \theta\right)\left(\theta \gamma_{\mu \nu \rho} \theta\right)$ & $c^{*}$ & $\left(\lambda \gamma^{\mu} \theta\right)\left(\lambda \gamma^{\nu} \theta\right)\left(\lambda \gamma^{\rho} \theta\right)\left(\theta \gamma_{\mu \nu \rho} \theta\right)$ & $\widetilde{c}^{p}$ \\
\hline
\end{tabular}

on the cohomologies of operator $Q=\lambda^{\alpha} \frac{\partial}{\partial \theta^{\alpha}}$. Here we use the following notations

$$
Q_{B}=\lambda^{\alpha} \frac{\partial}{\partial \theta^{\alpha}}+\frac{1}{2} \theta^{\alpha} \frac{\partial f^{\mu}}{\partial \lambda^{\alpha}} \partial_{\mu}, \quad \varepsilon^{\alpha} Q_{\alpha}^{s}=\varepsilon^{\alpha} \frac{\partial}{\partial \theta^{\alpha}}-\left(\varepsilon \gamma^{\mu} \theta\right) \frac{\partial}{\partial x^{\mu}}, \quad P_{\mu}^{s}=\frac{\partial}{\partial x^{\mu}}
$$

This calculation is done through the summation of all possible Feynman diagrams according to the standard rules discussed in section 4 (see also [2]). The degrees of freedom (representatives of $Q$-cohomologies) for this model are given by

The first and the third columns contain the polarizations (representatives of cohomologies) for the fields and antifields respectively, the second column gives the component fields of the superfield $A$ and the fourth one the component fields of the superfield $\mathrm{P}$. The component fields in the fourth column are BV antifields to the component fields in the second column. For example $\widetilde{c}$ is BV antifield to $c$. The same is true for other fields. Let $\left\{e_{B}\right\}$ denote the basis in the space of functions of $\lambda$ and $\theta$. Let $\left\{\underline{e}^{A}\right\}$ denote the dual basis in the dual space. There is a canonical pairing among them, that we denote as $\langle\rangle:,\left\langle\underline{e}^{A}, e_{B}\right\rangle=\delta_{B}^{A}$. For example

$$
<\underline{1}, 1>=1, \quad<\underline{1},\left(\lambda \gamma^{\mu} \theta\right)>=0
$$

\footnotetext{
${ }^{2}$ The problem of off-shell formulation in the context of harmonic superspace was studied in [16].
} 


\subsection{Doubling}

From the table (45) we see that the number of fields and anti-fields in the theory is twice the number of the fields we expect to have in SYM. The second column contains all the fields needed for BV version of SYM (this is true if one can think about the fields with the star-sign as antifields for the corresponding fields). In addition to them there are their BV antifields marked by tilde-sign (the content of the fourth column). At the present moment BV bracket is simply the canonical pairing, between $\mathrm{P}$ and $\mathrm{A}$.

We will call this theory, effective for (43), the pre-SYM. This funny name is due to the fact that the pre-theory and the theory (SYM) are related by the simple transformation which will be discussed in section 5.4 below. Roughly speaking, in order to get SYM itself one should identify some fields and antifields of the effective pre-theory as it is shown in the table (55).

\subsection{Technical Subtleties in the Calculation of Pre-SYM Action}

In the calculation we follow the standard technique. However it is technically complicated to project on cohomologies of $Q$. Instead we implement the following procedure [11].

Consider the space of functions of the 3 -rd power in $\lambda$ and the 5 -th power in $\theta$. Consider the subspace of this space $V_{1}$ generated by two elements

$$
\begin{gathered}
\left(\lambda \gamma^{\mu} \theta\right)\left(\lambda \gamma^{\nu} \theta\right)\left(\lambda \gamma^{\rho} \theta\right)\left(\theta \gamma_{a b c} \theta\right) \\
\left(\lambda \gamma^{\mu \nu \rho} \theta\right)\left(\lambda \gamma_{p} \theta\right)\left(\lambda \gamma_{q} \theta\right)\left(\theta \gamma_{a b c} \theta\right)
\end{gathered}
$$

This space $V_{1}$ can be decomposed into the sum of irreducible representations. The only cohomology in this space is $h_{3,5}=\left(\lambda \gamma^{\mu} \theta\right)\left(\lambda \gamma^{\nu} \theta\right)\left(\lambda \gamma^{\rho} \theta\right)\left(\theta \gamma_{\mu \nu \rho} \theta\right)$, which is a scalar. Consider linear functional «》 on the space $V_{1}$ such that it maps cohomology $h_{3,5}$ to 1 and non-trivial representations to zero. Namely [11]

$$
\begin{array}{r}
\ll\left(\lambda \gamma^{\mu} \theta\right)\left(\lambda \gamma^{\nu} \theta\right)\left(\lambda \gamma^{\rho} \theta\right)\left(\theta \gamma_{a b c} \theta\right) \gg=\frac{1}{120} \delta_{a b c}^{\mu \nu \rho} \\
\ll\left(\lambda \gamma^{\mu \nu \rho} \theta\right)\left(\lambda \gamma_{p} \theta\right)\left(\lambda \gamma_{q} \theta\right)\left(\theta \gamma_{a b c} \theta\right) \gg=\frac{1}{70} \delta_{[p}^{[\mu} \eta_{q][a} \delta_{b}^{\nu} \delta_{c]}^{\rho]}
\end{array}
$$

The coefficient is restored from the condition that the cohomology $h_{3,5}$ is mapped to unity.

In the computations that we perform we replace projection to cohomologies by the following procedure. For each representative $h_{a}$ from the table (45) define a complementary representative $d_{b}$ such that ${ }^{3}$

$$
\ll h_{a} \cdot d_{b} \gg=\delta_{a b}
$$

Here the product is induced by the multiplication of functions of $\lambda$ and $\theta$. To project some expression $\Omega$ onto the representative $h$ one should instead calculate the product with the complementary representative $d$

$$
\left.\Omega\right|_{h_{a}}=(-1)^{\# d_{a}+1} \ll d_{a} \cdot \Omega \gg
$$

Here $\# d_{a}$ denotes the parity of the representative $d_{a}$. It happens that with our choice of representatives and $K$ the result before projection is always in the space $V_{1}$ for all the Feynman diagrams.

Using this prescription one can calculate the effective action for the theory (43). The result is given by

$$
\begin{array}{r}
L_{p r e}^{e f f}=-\frac{1}{360} \widetilde{A}_{\mu}^{*} D_{\nu} F_{\mu \nu}+\frac{1}{160} \psi \gamma^{\mu} D_{\mu} \widetilde{\psi}^{*}+\frac{g}{160} \widetilde{A}_{\mu}^{*}\left(\psi \gamma^{\mu} \psi\right)+\widetilde{A}_{\mu} D_{\mu} c+A_{\mu}^{*} D_{\mu} \widetilde{c}^{*}-g A_{\mu}^{*}\left[\widetilde{A}_{\mu}^{*}, c\right]+ \\
+g\left(\widetilde{c} c c+\widetilde{c}^{*}\left[c^{*}, c\right]+\left[\widetilde{\psi}^{*}, \psi^{*}\right] c+[\widetilde{\psi}, \psi] c+\widetilde{c}^{*}\left[\psi^{*}, \psi\right]\right)+ \\
+\frac{3}{2}\left(\varepsilon \gamma^{\mu} \psi\right) \widetilde{A}_{\mu}+\frac{3}{2}\left(\varepsilon \gamma^{\mu} \widetilde{\psi}^{*}\right) A_{\mu}^{*}+\frac{2}{3}\left(\varepsilon \gamma^{\mu \nu} \widetilde{\psi}\right) D_{\mu} A_{\nu}+\frac{2}{3}\left(\varepsilon \gamma^{\mu \nu} \psi^{*}\right) D_{\mu} \widetilde{A}_{\nu}^{*}-\frac{g}{3}\left(\varepsilon \gamma^{\mu \nu} \widetilde{\psi}\right)\left[A_{\mu}, A_{\nu}\right]+ \\
+\eta^{\mu}\left[\widetilde{c} \partial_{\mu} c-c^{*} \partial_{\mu} \widetilde{c}^{*}+\widetilde{A}_{\nu} \partial_{\mu} A_{\nu}-A_{\nu}^{*} \partial_{\mu} \widetilde{A}_{\nu}^{*}+\left(\widetilde{\psi} \partial_{\mu} \psi\right)-\left(\psi^{*} \partial_{\mu} \widetilde{\psi}^{*}\right)\right]-\eta_{\mu}^{*}\left(\varepsilon \gamma^{\mu} \varepsilon\right)- \\
-80\left(\varepsilon \gamma^{\mu} \varepsilon\right)\left(\widetilde{\psi} \gamma_{\mu} \psi^{*}\right)+160(\varepsilon \widetilde{\psi})\left(\varepsilon \psi^{*}\right)-c^{*}\left(\varepsilon \gamma^{\mu} \varepsilon\right) \widetilde{A}_{\mu}^{*}-\widetilde{c}\left(\varepsilon \gamma^{\mu} \varepsilon\right) A_{\mu}
\end{array}
$$

The details of this calculation can be found in the appendix B.

Here $D_{\mu}=\partial_{\mu}+g\left[A_{\mu}, \cdot\right], F_{\mu \nu}=\partial_{\mu} A_{\nu}-\partial_{\nu} A_{\mu}+g\left[A_{\mu}, A_{\nu}\right]$.

We would like to emphasize that construction [11] with the bracket $\ll \gg$ is nothing but only a technical simplification in the way to project onto cohomologies.

\footnotetext{
${ }^{3}$ Since the bracket $\ll \gg$ maps all functions having the degree in $\lambda$ and $\theta$ different from 3 and 5 to zero, such complementary representative is unique and completes the degree in $\lambda$ and $\theta$ to 3,5 .
} 


\section{$5.3 \quad Z_{2}$ Duality of Feynman Diagrams}

The correspondence between the representatives (47) defines the $Z_{2}$ symmetry on cohomologies (the $Z_{2}$ symmetric representative is the one which completes the given one to non-vanishing value of the bracket $\ll$ ). Though this $Z_{2}$ symmetry on representatives is explicit (as is obvious from the table 45 and was discussed in [14]) it is unclear why it should be inherited by diagrams calculations. As a kind of experimental evidence of this fact below (fig.5) we present the results for several diagrams arising in the calculation of effective action at the quadratic level. From this figure it is clear that the vertices of the effective theory are symmetric w.r.t. the discussed $Z_{2}$ duality.
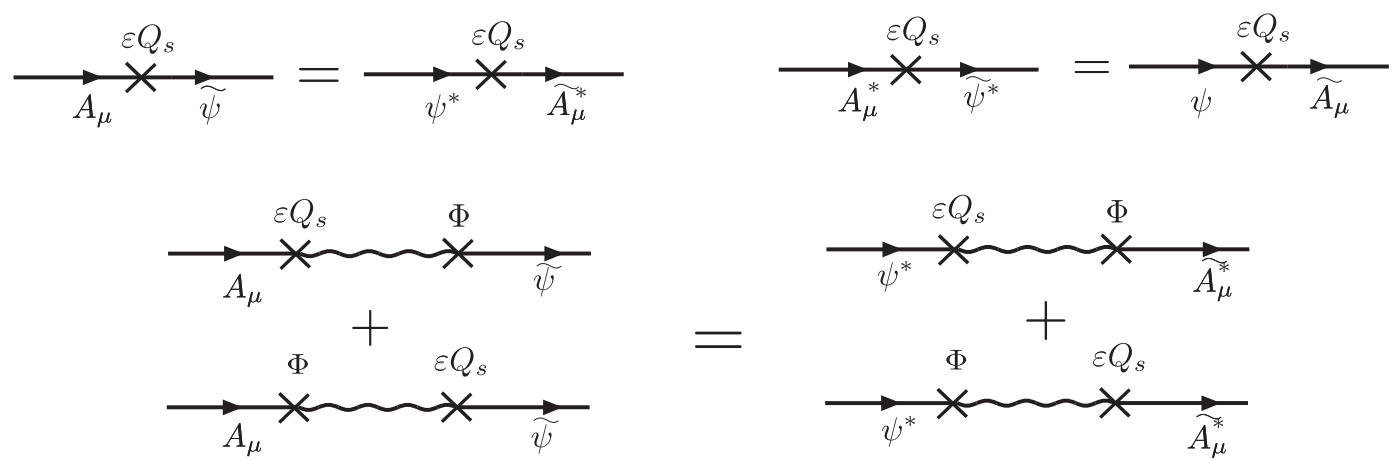

Figure 5: An example of $Z_{2}$ duality. Explicit calculations are presented in the appendix B.

One comment is in order here. Since we are interested in the action of SYM (not pre-SYM) we will finally identify the component fields of $\mathrm{A}$ and $\mathrm{P}$ according to (55). Mnemonic rule is the following: tilde and star is the same and tilde annihilates star. To understand the equalities in the figure 5 correctly one should make these identifications (55). The same is true for figure 6.

Note that these equalities express a non trivial statement, because incoming and the out-coming lines of the diagrams are completely different. All the operators are acting on the incoming lines and the result is projected on the out-coming line. Moreover, among these operators there are derivatives w.r.t $\theta$ (in $\varepsilon^{\alpha} Q_{\alpha}^{s}$ ). This duality states that there is, in a sense, a symmetry between the incoming and out-coming arrows. This symmetry results in the symmetry of the vertices of the effective action after the identification (55).

Duality rule. For generic subset of diagrams having certain external legs one can exchange the out-coming line with any of the in-lines simultaneously changing the star and tilde signs and shifting external legs in the cyclic way as it is shown in the fig. 6. The result does not change. It is important to remember that before application of this duality one should sum up all the diagrams having certain external legs (like in case of the last diagrams in the figure 5).
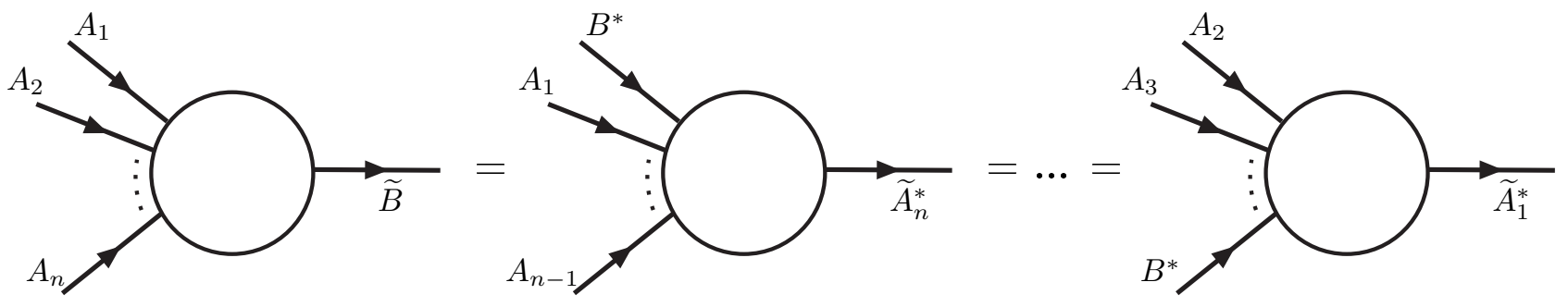

Figure 6: The $Z_{2}$ duality rule.

Sometimes this duality looks highly non-trivial at the diagram level and requires a lot of $\gamma$-matrix algebra to convince that the two contribution are indeed equal. This fact is completely obvious from the calculations presented in appendix B.

\subsection{The $Z_{2}$ projection}

After evaluation of effective action (pre-theory) it can be written in the following compact form

$$
S_{\text {pre }}^{\text {eff }}=P_{a} V^{a}(A)
$$


Here $P_{a}$ denote the components of superfield $\mathrm{P}$ and $V^{a}(A)$ is a vector field of the components $A^{a}$ of the superfield A, ghosts for SUSY and translations, and space-time derivatives. Since the action (50) is obtained via the integration over a lagrangian submanifold of BV action, it should satisfy classical BV equation, which can be written as $^{4}$

$$
P_{b} V^{a} \partial_{a} V^{b}=0
$$

In the previous section we discussed the $Z_{2}$ symmetry of the effective vertices, which was discovered experimentally at the level of Feynman diagrams calculation. Such symmetry in this compact notations can be written as

$$
\partial_{b} V^{a}(A) \eta_{a c}=\partial_{c} V^{a}(A) \eta_{a b}
$$

where $\eta_{a b}$ is non-degenerate pairing on cohomologies identifying the components of A and $\mathrm{P}$ via $P_{a}=\eta_{a b} A^{b}$ (see 55). This condition states that the vertices in the effective theory are symmetric w.r.t. the interchange of the fields of $\mathrm{A}$ and $\mathrm{P}$ at the external legs consistent with the $Z_{2}$ symmetry of representatives.

Relation (52) implies that $V^{a}$ can be written as a gradient

$$
V^{a}(A)=\eta^{a c} \partial_{c} \mathcal{F}
$$

Substituting this solution into BV equation (51)

$$
\partial_{d}\left(\eta^{a c} \partial_{a} \mathcal{F} \partial_{c} \mathcal{F}\right)=0
$$

In our calculations the constant of integration can be chosen to be zero and we come to the conclusion that function $\mathcal{F}$ satisfies classical BV equation on the space of $A$, namely

$$
\eta^{a b} \partial_{a} \mathcal{F} \partial_{b} \mathcal{F}=0 .
$$

The $\mathrm{BV}$ form in this equation coincides with the pairing $\eta_{a b}$ dictated by the $Z_{2}$ duality on representatives. This function $\mathcal{F}$ will play the role of BV action, which now depends only on the component fields of superfield A. Calculation of the function $\mathcal{F}$ for the pre-action (49) gives exactly SYM theory coupled to SUSY ghosts.

$$
\begin{array}{r}
S^{e f f}=\int \operatorname{Tr}\left(-\frac{1}{1440} F_{\mu \nu}^{2}-\frac{1}{320} \psi \gamma^{\mu} D_{\mu} \psi-A_{\rho}^{*} D_{\rho} c+g c^{*} c c+g\left[\psi^{*}, \psi\right] c-\right. \\
-\frac{3}{2}\left(\varepsilon \gamma^{\mu} \psi\right) A_{\mu}^{*}+\frac{1}{3}\left(\varepsilon \gamma^{\mu \nu} \psi^{*}\right) F_{\mu \nu}+\eta^{\mu}\left[c^{*} \partial_{\mu} c-A_{\nu}^{*} \partial_{\mu} A_{\nu}+\left(\psi^{*} \partial_{\mu} \psi\right)\right]-\eta_{\mu}^{*}\left(\varepsilon \gamma^{\mu} \varepsilon\right)- \\
\left.-40\left(\varepsilon \gamma^{\mu} \varepsilon\right)\left(\psi^{*} \gamma_{\mu} \psi^{*}\right)+80\left(\varepsilon \psi^{*}\right)^{2}-c^{*}\left(\varepsilon \gamma^{\mu} \varepsilon\right) A_{\mu}\right)
\end{array}
$$

\begin{tabular}{|c|c|c|c|c|}
\hline Polarization & $A$ & $P$ & & \\
\hline $\begin{array}{c}1 \\
\left(\lambda \gamma^{\mu} \theta\right)\end{array}$ & & & & $\begin{array}{c}\widetilde{c}=c^{*} \\
\widetilde{A}_{\mu}=-A_{\mu}^{*}\end{array}$ \\
\hline$\left(\lambda \gamma^{\mu} \theta\right)\left(\theta \gamma^{\mu}\right)_{\alpha}$ & & & $\Rightarrow$ & $\tilde{\bar{\psi}}^{\alpha}=\psi^{\alpha *}$ \\
\hline$-16\left(\lambda \gamma^{\mu} \theta\right)\left(\lambda \gamma^{\nu} \theta\right)\left(\theta \gamma^{\mu \nu}\right)^{\alpha}$ & & & & $-\psi_{\alpha}$ \\
\hline $\begin{array}{c}10\left(\lambda \gamma^{\mu} \theta\right)\left(\lambda \gamma^{\nu} \theta\right)\left(\theta \gamma_{\mu \nu \rho} \theta\right) \\
\left(\lambda \gamma^{\mu} \theta\right)\left(\lambda \gamma^{\nu} \theta\right)\left(\lambda \gamma^{\rho} \theta\right)\left(\theta \gamma_{\mu \nu \rho} \theta\right)\end{array}$ & & & & $\begin{array}{l}\widetilde{A}_{\rho}^{*}=A_{\rho} \\
\widetilde{c}^{*}=-c\end{array}$ \\
\hline
\end{tabular}

It is straightforward to check that (54) satisfy BV equation. It should be mentioned that at the linear level (coupling constant $g=0$ ), polynomial $V^{a}(A)$ have a certain degree of homogeneity (linear in the fields $A^{a}$ ). Hence, the integration (53) needed to extract action $\mathcal{F}$ from $V^{a}(A)$ results simply in the factor $\frac{1}{2}$. Thus at the linear level to obtain the action of the theory from the action of the pre-theory one should simply identify the component fields of $\mathrm{A}$ and $\mathrm{P}$ according to the following rule.

This rule determines the pairing $\eta_{a b}$.

\footnotetext{
${ }^{4}$ Solutions of this equation determine the so-called $\infty$-structure. In the context of quantum field theories on simplicial complexes these structures were recently studied in [8].
} 


\subsubsection{Naive $Z_{2}$ projection}

Naively, one could expect that just inverting the lines in the diagrams using the pairing $\eta_{a b}$ would be enough to produce the BV action of SYM. This naive procedure corresponds to

$$
S^{\text {Naive }}=\eta_{a b} A^{a} V^{b}(A)
$$

This however does not solve BV equation. In particular this will result in the fact that kinetic term for a gauge field would differ from $\operatorname{Tr} F_{\mu \nu}^{2}$. This can be checked by explicit calculation of the diagrams (see (94),(95) and (96) in appendix B). The final result for them after the identification (55) can be written as

$$
L=-\frac{1}{360} A_{\mu} D_{\nu} F_{\mu \nu}=-\frac{1}{1440}\left(\mathbf{2} \cdot\left(\partial_{\mu} A_{\nu}-\partial_{\nu} A_{\mu}\right)^{2}+\mathbf{3} \cdot 2 g\left(\partial_{\mu} A_{\nu}-\partial_{\nu} A_{\mu}\right)\left[A_{\mu}, A_{\nu}\right]+\mathbf{4} \cdot g^{2}\left[A_{\mu}, A_{\nu}\right]^{2}\right)
$$

This expression clarifies that to obtain correct result $\operatorname{Tr} F_{\mu \nu}^{2}$ one should put the coefficients $\frac{1}{2}, \frac{1}{3}$ and $\frac{1}{4}$ in front of the quadratic, cubic and quartic terms respectively. Remarkably that exactly these coefficients are dictated by the procedure (53). Note that this result can not be achieved by rescaling of the coupling constant $g \rightarrow 2 / 3 g$.

One of the main message of this last section is that the descent of a symmetry (for instance SUSY) from the action (1) down to (54) can be realized through the two steps:

1. One should calculate the path integral in the background of the cohomologies of $Q$ and find the pre-action. It is important that the action (1) is the off-shell supersymmetric version of pre-action. This descent was discussed in details in the previous section on the example of the 2- $d$ gauge model.

2. One should implement the procedure (53) to pass from the pre-action to an action. For the moment this step can not be done through the path integral. However, what can be said is that after the application of the second step the result satisfy classical BV equation over all the fields including ghosts for SUSY. Hence, some information about the off-shell description of SUSY in inherited in the action (54).

\section{Acknowledgments}

It is a pleasure to thank N. Berkovits, S. Demidov, E. Ivanov, C. Mafra, A. Morozov, V. Rubakov, M. Vasiliev and all the participants of the Workshop on Pure Spinors (Sao-Paulo, 2006) for useful discussions. Especially we would like to thank Chris Hull for pointing out the reference [7] to us. We are greatly indebted to Ulf Gran for providing us the version of his GAMMA package. This allowed us to speed up considerably the calculations with gamma matrices for the 10-dimensional Yang-Mills theory. DK and AL would like to thank the organizers of the Workshop on Pure Spinors (Sao-Paulo, 2006) for hospitality and creation of the stimulating atmosphere in which a part of this work had been done. The work of DK was supported by the grant RFBR-04-02-17227, the grant of the President of the Russian Federation NS-7293.2006.2 (government contract 02.445.11.7370) and the fellowship of Dynasty Foundation in 2007. The work of AL was supported by the grant RFBR 04-0217227, INTAS 03-51-6346 and the grant for support of scientific schools NSh-8065.2006.2. The work of VL was supported by the grant RFBR 04-02-16538 and INTAS 03-51-6346. 


\section{A Appendix. Some properties of $S O(10) \gamma$ - matrices}

In this appendix we summarize some properties of $S O(10) \gamma$-matrices, which are important for our calculations. Another list of useful identities can be found in the appendix of [11]. Through the whole paper we do not distinguish between upper and lower $S O(10)$ vector indices and use the convention, that $\gamma^{\mu_{1} \ldots \mu_{n}}=\frac{1}{n !} \gamma^{\left[\mu_{1}\right.} \ldots \gamma^{\left.\mu_{n}\right]}$.

Ten dimensional $\gamma$ - matrices can have two upper spinor indices $\left(\gamma^{\mu}\right)^{\alpha \beta}$ or two lower indices $\left(\gamma^{\mu}\right)_{\alpha \beta}$. Both these two matrices are symmetric in $\alpha$ and $\beta$.

Using this convention it is straightforward to check the symmetry properties of the following representations

\begin{tabular}{|c|c|}
\hline Symmetric & Antisymmetric \\
\hline $\begin{array}{c}\left(\gamma^{\mu}\right)_{\alpha \beta} \quad\left(\gamma^{\mu_{1} \ldots \mu_{4}}\right)_{\alpha}^{\beta} \quad\left(\gamma^{\mu_{1} \ldots \mu_{5}}\right)_{\alpha \beta} \\
\left(\gamma^{\mu_{1} \ldots \mu_{8}}\right)_{\alpha}^{\beta}\left(\gamma^{\mu_{1} \ldots \mu_{9}}\right)_{\alpha \beta}\end{array}$ & $\left(\gamma^{\mu_{1} \ldots \mu_{6}}\right)_{\alpha}{ }^{\left.\beta^{\left(\gamma^{\mu \nu}\right.}\right)_{\alpha^{\beta}}\left(\gamma^{\mu_{1} \ldots \mu_{7}}\right)_{\alpha \beta}\left(\gamma^{\mu \nu \rho}\right)_{\alpha \beta}}\left(\gamma^{\mu_{1} \ldots \mu_{10}}\right)_{\alpha}{ }^{\beta}$ \\
\hline
\end{tabular}

For the representations with even number of $\gamma$-matrices (which hence have one upper and one lower spinor index) by symmetry properties we mean $A_{\alpha}{ }^{\beta}= \pm A^{\beta}{ }_{\alpha}$

Due to duality properties of $\gamma$-matrices

$$
\gamma^{\mu_{1} \ldots \mu_{n}}= \pm \frac{1}{(10-n) !} \varepsilon^{\mu_{1} \ldots \mu_{10}} \gamma_{\mu_{n+1} \ldots \mu_{10}}
$$

the basis in the space of all matrices is given by

$$
\delta_{\alpha}^{\beta}, \quad\left(\gamma^{\mu}\right)_{\alpha \beta}, \quad\left(\gamma^{\mu}\right)^{\alpha \beta}, \quad\left(\gamma^{\mu \nu}\right)_{\alpha}^{\beta}, \quad\left(\gamma^{\mu \nu \rho}\right)_{\alpha \beta}, \quad\left(\gamma^{\mu \nu \rho}\right)^{\alpha \beta}, \quad\left(\gamma^{\mu_{1} \ldots \mu_{4}}\right)_{\alpha}^{\beta}, \quad\left(\gamma^{\mu_{1} \ldots \mu_{5}}\right)_{\alpha \beta}, \quad\left(\gamma^{\mu_{1} \ldots \mu_{5}}\right)^{\alpha \beta} .
$$

The system (58) is complete. This fact allows to prove certain Fiertz identities. To illustrate the procedure consider the following identity

$$
\left(\gamma^{\mu}\right)_{\alpha \beta}\left(\gamma_{\mu}\right)_{\delta \sigma}=-\frac{1}{2}\left(\gamma^{a}\right)_{\alpha \delta}\left(\gamma_{a}\right)_{\beta \sigma}-\frac{1}{24}\left(\gamma^{a b c}\right)_{\alpha \delta}\left(\gamma_{a b c}\right)_{\beta \sigma} .
$$

Expanding the 1.h.s. in the complete set (58) with respect to the indices $\alpha$ and $\delta$ one can write

$$
\left(\gamma^{\mu}\right)_{\alpha \beta}\left(\gamma_{\mu}\right)_{\delta \sigma}=C_{1}\left(\gamma^{a}\right)_{\alpha \delta}\left(\gamma_{a}\right)_{\beta \sigma}+C_{3}\left(\gamma^{a b c}\right)_{\alpha \delta}\left(\gamma_{a b c}\right)_{\beta \sigma}+C_{5}\left(\gamma^{a b c d e}\right)_{\alpha \delta}\left(\gamma_{a b c d e}\right)_{\beta \sigma}
$$

we can use only combinations with odd number of $\gamma$ - matrices because all spinor indices are lower. Multiplying both sides of relation (60) separately by $\left(\gamma^{\nu}\right)^{\beta \delta},\left(\gamma^{\nu_{1} \nu_{2} \nu_{3}}\right)^{\beta \delta}$ and $\left(\gamma^{\nu_{1} \ldots \nu_{5}}\right)^{\beta \delta}$ and using the identities like

$$
\begin{aligned}
& \gamma^{a} \gamma^{\nu} \gamma_{a}=-8 \gamma^{\nu}, \\
& \gamma^{a} \gamma^{\mu \nu \rho} \gamma_{a}=-4 \gamma^{\mu \nu \rho}, \\
& \gamma^{a b c} \gamma^{\mu} \gamma_{a b c}=288 \gamma^{\mu}, \\
& \gamma^{a b c} \gamma^{\mu \nu \rho} \gamma_{a b c}=-48 \gamma^{\mu \nu \rho}, \\
& \gamma^{a b c} \gamma^{\mu \ldots \mu_{5}} \gamma_{a b c}=0, \\
& \gamma^{a_{1} \ldots a_{5}} \gamma^{\mu} \gamma_{a_{1} \ldots a_{5}}=\gamma^{a_{1} \ldots a_{5}} \gamma^{\mu \nu \rho} \gamma_{a_{1} \ldots a_{5}}=\gamma^{a_{1} \ldots a_{5}} \gamma^{\mu_{1} \ldots \mu_{5}} \gamma_{a_{1} \ldots a_{5}}=0
\end{aligned}
$$

one can straightforwardly fix the coefficients $C_{1}, C_{3}$ and $C_{5}$ in (60). In this calculation it is important to remember the symmetry properties mentioned in the table (56). Relations (61) can be derived using the definition of $\gamma$-matrices: $\left\{\gamma^{\mu}, \gamma^{\nu}\right\}=2 g^{\mu \nu}$. We acknowledge inestimable help of Ulf Gran's GAMMA package [12] in doing these computations.

Similar technique allows to obtain other Fiertz identities, for example

$$
\left(\gamma^{\mu \nu \rho}\right)_{\alpha \beta}\left(\gamma_{\mu \nu \rho}\right)_{\delta \sigma}=-18\left(\gamma^{a}\right)_{\alpha \delta}\left(\gamma_{a}\right)_{\beta \sigma}+\frac{1}{2}\left(\gamma^{a b c}\right)_{\alpha \delta}\left(\gamma_{a b c}\right)_{\beta \sigma}
$$

Independent check that expressions (59) and (62) are consistent can be done by double expansion of l.h.s of (59): firstly in the indices $\alpha, \delta$, secondly in the indices $\alpha, \beta$. Making this double expansion one should come in the end to the initial expression $\left(\gamma^{\mu}\right)_{\alpha \beta}\left(\gamma_{\mu}\right)_{\delta \sigma}$.

Applying the same machinery it is straightforward to prove another useful identity

$$
\left(\gamma^{\mu_{1} \ldots \mu_{4}}\right)_{\alpha}{ }^{\beta}\left(\gamma_{\mu_{1} \ldots \mu_{4}}\right)_{\sigma}{ }^{\delta}=315 \delta_{\alpha}{ }^{\delta} \delta_{\sigma}{ }^{\beta}+\frac{21}{2}\left(\gamma^{a b}\right)_{\alpha}{ }^{\delta}\left(\gamma_{a b}\right)_{\sigma}{ }^{\beta}+\frac{1}{8}\left(\gamma^{a_{1} \ldots a_{4}}\right)_{\alpha}{ }^{\delta}\left(\gamma_{a_{1} \ldots a_{4}}\right)_{\sigma}{ }^{\beta}
$$


Application of relation (59) allows to prove useful identity

$$
\left(\lambda \gamma^{\mu} \psi\right)\left(\lambda \gamma_{\mu} \xi\right)=0, \quad \forall \psi^{\alpha}, \xi^{\alpha},
$$

mentioned in the appendix to [11]. Indeed, applying (59) one can find

$$
\left(\lambda \gamma^{\mu} \psi\right)\left(\lambda \gamma_{\mu} \xi\right)=-\frac{1}{2}\left(\lambda \gamma^{\mu} \lambda\right)\left(\psi \gamma_{\mu} \xi\right)-\frac{1}{24}\left(\lambda \gamma^{a b c} \lambda\right)\left(\psi \gamma_{a b c} \xi\right)=0 .
$$

The first term is equal to zero due to pure spinor constrains $\left(\lambda \gamma^{\mu} \lambda\right)=0$, the second one is equal to zero because $\left(\gamma^{a b c}\right)_{\alpha \beta}$ is antisymmetric in $\alpha, \beta$ (see table 56) while combination $\lambda^{\alpha} \lambda^{\beta}$ is symmetric.

Sometimes it is useful to have the representation for antisymmetric bi-spinor

$$
\theta^{\alpha} \theta^{\beta}=\frac{1}{96}\left(\theta \gamma^{a b c} \theta\right)\left(\gamma_{a b c}\right)^{\alpha \beta}
$$

These relations can be derived in a similar way: for generic bi-spinor one can write an expansion

$$
\xi^{\alpha} \psi^{\beta}=\frac{1}{16}\left(\xi \gamma^{\mu} \psi\right)\left(\gamma_{\mu}\right)^{\alpha \beta}+\frac{1}{96}\left(\xi \gamma^{a b c} \psi\right)\left(\gamma_{a b c}\right)^{\alpha \beta}+\frac{1}{3840}\left(\xi \gamma^{\mu_{1} \ldots \mu_{5}} \psi\right)\left(\gamma_{\mu_{1} \ldots \mu_{5}}\right)^{\alpha \beta} .
$$

The coefficients in this expression can be determined by contracting both hand sides with $\left(\gamma^{\mu}\right)_{\alpha \beta},\left(\gamma^{\mu \nu \rho}\right)_{\alpha \beta}$ and $\left(\gamma^{\mu_{1} \ldots \mu_{5}}\right)_{\alpha \beta}$. In case of antisymmetric bi-spinor $\theta \gamma^{\mu} \theta=0$ and $\theta \gamma^{\mu_{1} \ldots \mu_{5}} \theta=0$ because these expressions are symmetric in spinor indices. The representation for symmetric pure bi-spinor gives ${ }^{5}$

$$
\lambda^{\alpha} \lambda^{\beta}=\frac{1}{3840}\left(\lambda \gamma^{\mu_{1} \ldots \mu_{5}} \lambda\right)\left(\gamma_{\mu_{1} \ldots \mu_{5}}\right)^{\alpha \beta}
$$

due to constrains $\left(\lambda \gamma^{\mu} \lambda\right)=0$.

In our calculations we will also need a representation [11]

$$
\left(\lambda \gamma^{\mu} \theta\right)\left(\lambda \gamma^{\nu} \theta\right)=\frac{1}{2}\left(\lambda \gamma^{a \mu \nu} \theta\right)\left(\lambda \gamma_{a} \theta\right)
$$

which can be proven by applying formula (59) and commutation relations $\left\{\gamma^{\mu}, \gamma^{\nu}\right\}=2 g^{\mu \nu}$ in the r.h.s.

One more useful formula [13] is

$$
\left(\gamma^{\mu \nu}\right)_{\alpha}{ }^{\delta}\left(\gamma_{\mu \nu}\right)_{\beta}{ }^{\sigma}=-8 \delta_{\alpha}{ }^{\sigma} \delta_{\beta}{ }^{\delta}+4\left(\gamma^{\mu}\right)_{\alpha \beta}\left(\gamma_{\mu}\right)^{\delta \sigma}-2 \delta_{\alpha}{ }^{\delta} \delta_{\beta}{ }^{\sigma}
$$

In our calculations we also use scalar ${ }^{6}$ product [11]

$$
\begin{array}{r}
\ll\left(\lambda \gamma^{\mu} \theta\right)\left(\lambda \gamma^{\nu} \theta\right)\left(\lambda \gamma^{\rho} \theta\right)\left(\theta \gamma_{a b c} \theta\right) \gg=\frac{1}{120} \delta_{a b c}^{\mu \nu \rho} \\
\ll\left(\lambda \gamma^{\mu \nu \rho} \theta\right)\left(\lambda \gamma_{p} \theta\right)\left(\lambda \gamma_{q} \theta\right)\left(\theta \gamma_{a b c} \theta\right) \gg=\frac{1}{70} \delta_{[p}^{[\mu} \eta_{q][a} \delta_{b}^{\nu} \delta_{c]}^{\rho]}
\end{array}
$$

\section{B Appendix. Calculation of effective action for the $D=10$ SYM}

In this appendix we present the calculation of effective action [2] for the theory

$$
S^{S U S Y}=\int \operatorname{Tr}\left(<\mathcal{P}, Q_{B} \mathcal{A}>+g<\mathcal{P}, \mathcal{A}^{2}>+<\mathcal{P}, \varepsilon^{\alpha} Q_{\alpha}^{s} \mathcal{A}>+<\mathcal{P}, \eta^{\mu} P_{\mu}^{s} \mathcal{A}>-\eta_{\mu}^{*}\left(\varepsilon \gamma^{\mu} \varepsilon\right)\right)
$$

along the way described in section 5 , namely using the scalar product $\ll \gg$, defined in (70) and (71). Operators $Q_{B}, P_{s}$ and $Q_{s}$ are defined as

$$
Q_{B}=Q+\Phi=\lambda^{\alpha} \frac{\partial}{\partial \theta^{\alpha}}+\frac{1}{2} \theta^{\alpha} \frac{\partial f^{\mu}}{\partial \lambda^{\alpha}} \partial_{\mu}, \quad \varepsilon^{\alpha} Q_{\alpha}^{s}=\varepsilon^{\alpha} \frac{\partial}{\partial \theta^{\alpha}}-\left(\varepsilon \gamma^{\mu} \theta\right) \frac{\partial}{\partial x^{\mu}}, \quad P_{\mu}^{s}=\frac{\partial}{\partial x^{\mu}}
$$

Polarizations of component fields are given by (74) (see (45))

\footnotetext{
${ }^{5}$ We are indebted to Carlos Mafra for the correction of the coefficient in this expression.

${ }^{6} \delta_{a b c}^{\mu \nu \rho}=\frac{1}{3 !} \delta_{a}^{[\mu} \delta_{b}^{\nu} \delta_{c}^{\rho]}$
} 


\begin{tabular}{ccc}
\hline Polarization & $A$ & $P$ \\
\hline 1 & $c$ & $\widetilde{c}$ \\
$\left(\lambda \gamma^{\mu} \theta\right)$ & $A_{\mu}$ & $\widetilde{A}_{\mu}$ \\
$\left(\lambda \gamma^{\mu} \theta\right)\left(\theta \gamma^{\mu}\right)_{\alpha}$ & $\psi^{\alpha}$ & $\widetilde{\psi}^{\alpha}$ \\
$-16\left(\lambda \gamma^{\mu} \theta\right)\left(\lambda \gamma^{\nu} \theta\right)\left(\theta \gamma^{\mu \nu}\right)^{\alpha}$ & $\psi_{\alpha}^{*}$ & $\widetilde{\psi}_{\alpha}^{*}$ \\
$10\left(\lambda \gamma^{\mu} \theta\right)\left(\lambda \gamma^{\nu} \theta\right)\left(\theta \gamma_{\mu \nu \rho} \theta\right)$ & $A_{\rho}^{*}$ & $\widetilde{A}_{\rho}^{*}$ \\
$\left(\lambda \gamma^{\mu} \theta\right)\left(\lambda \gamma^{\nu} \theta\right)\left(\lambda \gamma^{\rho} \theta\right)\left(\theta \gamma_{\mu \nu \rho} \theta\right)$ & $c^{*}$ & $\widetilde{c}^{*}$
\end{tabular}

We start from the quadratic terms in the action and then derive interaction terms.

\section{Quadratic level}

First of all we evaluate the diagrams which do not depend on the ghosts $\varepsilon^{\alpha}$ and $\eta^{\mu}$. These diagrams contribute into the classical part of effective action. Below we list the nontrivial diagrams, intermediate and final expressions for them. The first diagram is

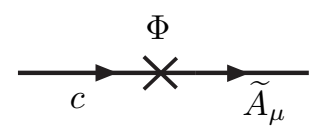

We emphasize that one should first act by all the operators and propagators onto the incoming field and then project the result onto cohomologies according the procedure discussed in section 5 . Namely, to project the result $R$ onto a representative $h$ corresponding to a certain component field $B$ one should calculate $\ll \gg$ with the complementary representative $d$, definition is given in (47). The contribution into the action will be given by $(-1)^{\# \widetilde{B}} \ll d \widetilde{B} \cdot R \gg$. Here $\# \widetilde{B}$ denotes the parity of the component field $\widetilde{B}$. The relative sign $(-1)^{\# \widetilde{B}}$ will be taken into account only in the final result (97).

The contribution of the first diagram is given by

$$
\mathcal{D}=\ll 10\left(\lambda \gamma_{\mu} \theta\right)\left(\lambda \gamma_{\nu} \theta\right)\left(\theta \gamma^{\mu \nu \rho} \theta\right) \widetilde{A}_{\rho} \cdot\left(\lambda \gamma_{a} \theta\right) \partial_{a} c \gg=-\widetilde{A}_{\rho} \frac{10}{120} \delta_{\mu \nu \rho}^{\mu \nu a} \partial_{a} c=-\widetilde{A}_{\rho} \partial_{\rho} c .
$$

Here we used the parities of component fields and the definition (70).

Dual diagram is

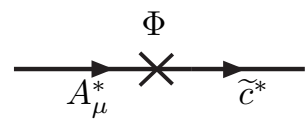

$$
\begin{aligned}
& \mathcal{D}=\ll \widetilde{c}^{*} \cdot\left(\lambda \gamma^{a} \theta\right) \partial_{a} \cdot 10\left(\lambda \gamma_{\mu} \theta\right)\left(\lambda \gamma_{\nu} \theta\right)\left(\theta \gamma^{\mu \nu \rho} \theta\right) A_{\rho}^{*} \gg=-\frac{10}{120} \widetilde{c}^{*} \delta_{\mu \nu \rho}^{\mu \nu a} \partial_{a} A_{\rho}^{*}=-A_{\rho}^{*} \partial_{\rho} \widetilde{c}^{*}
\end{aligned}
$$

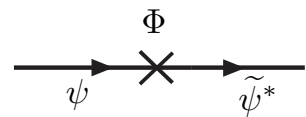

$$
\begin{aligned}
& \mathcal{D}=\ll\left(\lambda \gamma_{a} \theta\right)\left(\theta \gamma_{a} \widetilde{\psi}^{*}\right)\left(\lambda \gamma^{\nu} \theta\right)\left(\lambda \gamma^{\mu} \theta\right)\left(\theta \gamma^{\mu} \partial_{\nu} \psi\right) \gg=-\frac{1}{96} \ll\left(\lambda \gamma_{a} \theta\right)\left(\lambda \gamma_{\nu} \theta\right)\left(\lambda \gamma_{\mu} \theta\right)\left(\theta \gamma^{b c d} \theta\right) \gg \widetilde{\psi}^{*} \gamma^{a} \gamma_{b c d} \gamma^{\mu} \partial_{\nu} \psi= \\
& =-\frac{1}{96 \cdot 120} \widetilde{\psi}^{*} \gamma^{a} \gamma_{a \nu \mu} \gamma^{\mu} \partial_{\nu} \psi=-\frac{72}{96 \cdot 120} \widetilde{\psi}^{*} \gamma_{\nu} \partial_{\nu} \psi=-\frac{1}{160} \widetilde{\psi}^{*} \gamma_{\nu} \partial_{\nu} \psi
\end{aligned}
$$

Here we used (65) and the definition of $\gamma$ - matrices. This diagrams give kinetic term for the fermion.

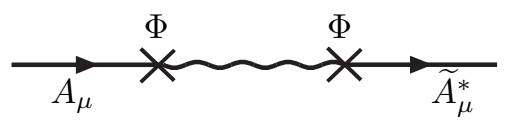


This diagram is responsible for the kinetic term for the gauge field. First of all we calculate the action of operator $\Phi$

$$
\Phi\left(\lambda \gamma^{\mu} \theta\right) A_{\mu}=\left(\lambda \gamma^{\mu} \theta\right)\left(\lambda \gamma^{\nu} \theta\right) \partial_{\mu} A_{\nu}=\frac{1}{2}\left(\lambda \gamma^{a \mu \nu} \theta\right)\left(\lambda \gamma_{a} \theta\right) \partial_{\mu} A_{\nu}
$$

In the last transformation we used (68). This should be done to prepare the result for application of the propagator (it is impossible to do it directly on $\left(\lambda \gamma^{\mu} \theta\right)\left(\lambda \gamma^{\nu} \theta\right)$ because $\left(\gamma^{\mu}\right)_{\alpha \beta}$ is symmetric w.r.t. $\alpha$ and $\beta$ ). The pre-mage is given by

$$
\Pi=\frac{1}{4}\left(\theta \gamma^{a \mu \nu} \theta\right)\left(\lambda \gamma_{a} \theta\right) \partial_{\mu} A_{\nu}
$$

Indeed, $Q \cdot \Pi=\lambda \frac{\partial}{\partial \theta} \Pi=\frac{1}{2}\left(\lambda \gamma^{a \mu \nu} \theta\right)\left(\lambda \gamma_{a} \theta\right) \partial_{\mu} A_{\nu}$ due to pure spinor constraints $\left(\lambda \gamma_{a} \lambda\right)=0$. The whole contribution of the diagram is given by

$$
\mathcal{D}=\ll \frac{1}{4}\left(\lambda \gamma_{\rho} \theta\right) \widetilde{A}_{\rho}^{*}\left(\lambda \gamma_{\varepsilon} \theta\right)\left(\theta \gamma^{a \mu \nu} \theta\right)\left(\lambda \gamma^{a} \theta\right) \gg \partial_{\varepsilon} \partial_{\mu} A_{\nu}=\frac{1}{4 \cdot 120} \delta_{a \mu \nu}^{\rho \varepsilon a} \widetilde{A}_{\rho}^{*} \partial_{\varepsilon} \partial_{\mu} A_{\nu}=-\frac{1}{360}\left(\widetilde{A}_{\mu}^{*} \partial^{2} A_{\mu}-\widetilde{A}_{\mu}^{*} \partial_{\mu} \partial_{\nu} A_{\nu}\right) .
$$

If one identifies $\widetilde{A}_{\mu}^{*}=A_{\mu}$ it is possible recognize in this result abelian part of $\frac{1}{720} F_{\mu \nu}^{2}$.

Now we switch to the calculation of diagrams proportional to the ghost for the SUSY. The first diagram is

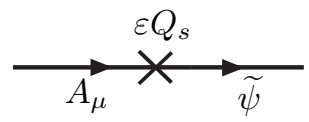

It is enough to use only the second part of $Q_{s}$, proportional to space-time derivative ${ }^{7}$

$$
\varepsilon Q_{s}\left(\lambda \gamma^{\mu} \theta\right) A_{\mu}=-\left(\varepsilon \gamma^{\nu} \theta\right)\left(\lambda \gamma^{\mu} \theta\right) \partial_{\nu} A_{\mu} .
$$

The contribution of the diagram is

$$
\begin{aligned}
\mathcal{D}=16 \ll\left(\lambda \gamma^{a} \theta\right) & \left(\lambda \gamma^{b} \theta\right)\left(\theta \gamma^{a b} \widetilde{\psi}\right)\left(\varepsilon \gamma^{\nu} \theta\right)\left(\lambda \gamma^{\mu} \theta\right) \gg \partial_{\nu} A_{\mu}=\frac{1}{6} \ll\left(\lambda \gamma^{a} \theta\right)\left(\lambda \gamma^{b} \theta\right)\left(\lambda \gamma^{\mu} \theta\right)\left(\theta \gamma^{m n k} \theta\right) \gg \times \\
& \times\left(\varepsilon \gamma^{\nu} \gamma^{m n k} \gamma^{b a} \widetilde{\psi}\right) \partial_{\nu} A_{\mu}=-\frac{1}{6 \cdot 120}\left(\varepsilon \gamma^{\nu} \gamma^{a b \mu} \gamma^{a b} \widetilde{\psi}\right) \partial_{\nu} A_{\mu}=\frac{1}{10}\left(\varepsilon \gamma^{\nu} \gamma^{\mu} \widetilde{\psi}\right) \partial_{\nu} A_{\mu} .
\end{aligned}
$$

The $Z_{2}$ dual diagram is

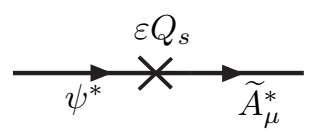

Application of supercharge to $\psi^{*}$ gives

$$
\varepsilon Q_{s}\left[-16\left(\lambda \gamma^{\mu} \theta\right)\left(\lambda \gamma^{\nu} \theta\right)\left(\theta \gamma^{\mu \nu} \psi^{*}\right)\right]=16\left(\varepsilon \gamma^{\rho} \theta\right)\left(\lambda \gamma^{\mu} \theta\right)\left(\lambda \gamma^{\nu} \theta\right)\left(\theta \gamma^{\mu \nu} \partial_{\rho} \psi^{*}\right)
$$

The contribution of the diagram is

$$
\mathcal{D}=16 \ll\left(\lambda \gamma^{\rho} \theta\right) \widetilde{A}_{\rho}^{*}\left(\varepsilon \gamma^{\lambda} \theta\right)\left(\lambda \gamma^{\mu} \theta\right)\left(\lambda \gamma^{\nu} \theta\right)\left(\theta \gamma^{\mu \nu} \partial_{\lambda} \psi^{*}\right) \gg=-\frac{1}{10}\left(\varepsilon \gamma^{\mu} \gamma^{\nu} \partial_{\mu} \psi^{*}\right) \widetilde{A}_{\nu}^{*}
$$

Figure 7:

In contrast to previous pair of diagrams in this case it is enough to use only the first term ${ }^{7}$ in $Q_{s}$ acting as derivative $\frac{\partial}{\partial \theta}$

$$
\varepsilon Q_{s}\left[\left(\lambda \gamma^{\mu} \theta\right)\left(\theta \gamma^{\mu} \psi\right)\right]=\left(\lambda \gamma^{\mu} \varepsilon\right)\left(\theta \gamma^{\mu} \psi\right)-\left(\lambda \gamma^{\mu} \theta\right)\left(\varepsilon \gamma^{\mu} \psi\right) .
$$

Using the formula (59) one can expand the contribution of the first term and obtain

\footnotetext{
${ }^{7}$ The other term of $Q_{s}$ does not contribute to the final result of the diagram due to the degree in $\lambda$ and $\theta$.
} 


$$
\begin{array}{r}
\mathcal{D}=10 \ll\left(\lambda \gamma^{a} \theta\right)\left(\lambda \gamma^{b} \theta\right)\left(\theta \gamma^{a b \rho} \theta\right) \widetilde{A}_{\rho}\left(\lambda \gamma^{\mu} \varepsilon\right)\left(\theta \gamma^{\mu} \psi\right) \gg-10 \ll\left(\lambda \gamma^{a} \theta\right)\left(\lambda \gamma^{b} \theta\right)\left(\theta \gamma^{a b \rho} \theta\right) \widetilde{A}_{\rho}\left(\lambda \gamma^{\mu} \theta\right) \gg\left(\varepsilon \gamma^{\mu} \psi\right)= \\
=-\frac{10}{2} \ll\left(\lambda \gamma^{a} \theta\right)\left(\lambda \gamma^{b} \theta\right)\left(\theta \gamma^{a b \rho} \theta\right)\left(\lambda \gamma^{\mu} \theta\right) \gg\left(\varepsilon \gamma^{\mu} \psi\right) \widetilde{A}_{\rho}-\frac{10}{24} \ll\left(\lambda \gamma^{a} \theta\right)\left(\lambda \gamma^{b} \theta\right)\left(\theta \gamma^{a b \rho} \theta\right)\left(\lambda \gamma^{\mu \nu c} \theta\right) \gg\left(\varepsilon \gamma^{\mu \nu c} \psi\right) \widetilde{A}_{\rho} \\
-10 \ll\left(\lambda \gamma^{a} \theta\right)\left(\lambda \gamma^{b} \theta\right)\left(\theta \gamma^{a b \rho} \theta\right)\left(\lambda \gamma^{\mu} \theta\right) \gg\left(\varepsilon \gamma^{\mu} \psi\right) \widetilde{A}_{\rho}=-\frac{10}{2 \cdot 120} \delta_{a b \rho}^{a b \mu}\left(\varepsilon \gamma^{\mu} \psi\right) \widetilde{A}_{\rho}- \\
-\frac{10}{120} \delta_{a b \rho}^{a b \mu}\left(\varepsilon \gamma^{\mu} \psi\right) \widetilde{A}_{\rho}-\frac{10}{24 \cdot 70} \delta_{[a}^{[\mu} \eta_{b][a} \delta_{b}^{\nu} \delta_{\rho]}^{c]}\left(\varepsilon \gamma_{\mu \nu c} \psi\right) \widetilde{A}_{\rho}=-\frac{3}{2}\left(\varepsilon \gamma_{\rho} \psi\right) \widetilde{A}_{\rho} .
\end{array}
$$

The last term with the coefficient $\frac{10}{24 \cdot 70}$ is equal to zero due to symmetry properties.

Calculation of dual diagram is more tricky.

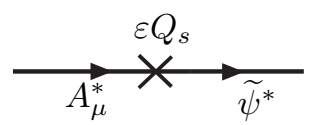

Figure 8:

Again operator $Q_{s}$ acts as derivative ${ }^{7} \frac{\partial}{\partial \theta}$

$$
\varepsilon Q_{s}\left[10\left(\lambda \gamma^{\mu} \theta\right)\left(\lambda \gamma^{\nu} \theta\right)\left(\theta \gamma^{\mu \nu \rho} \theta\right) A_{\rho}^{*}\right]=20\left(\lambda \gamma^{\mu} \varepsilon\right)\left(\lambda \gamma^{\nu} \theta\right)\left(\theta \gamma^{\mu \nu \rho} \theta\right) A_{\rho}^{*}+20\left(\lambda \gamma^{\mu} \theta\right)\left(\lambda \gamma^{\nu} \theta\right)\left(\varepsilon \gamma^{\mu \nu \rho} \theta\right) A_{\rho}^{*} .
$$

Evaluating the contribution of the first term one can find

$$
\begin{gathered}
\ll\left(\lambda \gamma^{a} \theta\right)\left(\theta \gamma^{a} \widetilde{\psi}^{*}\right)\left(\lambda \gamma^{\mu} \varepsilon\right)\left(\lambda \gamma^{\nu} \theta\right)\left(\theta \gamma^{\mu} \gamma^{\nu} \gamma^{\rho} \theta\right) \gg=-\frac{1}{2} \ll\left(\lambda \gamma^{a} \theta\right)\left(\theta \gamma^{a} \widetilde{\psi}^{*}\right)\left(\lambda \gamma^{\nu} \theta\right)\left(\lambda \gamma^{b} \theta\right)\left(\varepsilon \gamma^{b} \gamma^{\nu} \gamma^{\rho} \theta\right) \gg- \\
-\frac{1}{24} \ll\left(\lambda \gamma^{a} \theta\right)\left(\theta \gamma^{a} \widetilde{\psi}^{*}\right)\left(\lambda \gamma^{\nu} \theta\right)\left(\lambda \gamma^{m n p} \theta\right)\left(\varepsilon \gamma_{m n p} \gamma^{\nu} \gamma^{\rho} \theta\right) \gg=-\frac{1}{2 \cdot 96 \cdot 120}\left(\varepsilon \gamma^{b} \gamma^{\nu} \gamma^{\rho} \gamma_{a \nu b} \gamma^{a} \widetilde{\psi}^{*}\right)- \\
-\frac{1}{24 \cdot 96 \cdot 70} \delta_{[a}^{[m} \eta_{\varepsilon][p} \delta_{q}^{n} \delta_{c]}^{k]}\left(\varepsilon \gamma_{m n k} \gamma^{\varepsilon} \gamma^{\rho} \gamma^{p q c} \gamma^{a} \widetilde{\psi}^{*}\right)=\left(-\frac{432}{2 \cdot 96 \cdot 120}-\frac{1008}{24 \cdot 96 \cdot 70}\right)\left(\varepsilon \gamma^{\rho} \widetilde{\psi}^{*}\right)=-\frac{1}{40}\left(\varepsilon \gamma^{\rho} \widetilde{\psi}^{*}\right) .
\end{gathered}
$$

Here we used that $\theta \gamma^{\mu \nu \rho} \theta=\theta \gamma^{\mu} \gamma^{\nu} \gamma^{\rho} \theta$ (This is due to $\theta \gamma^{\mu} \theta=0$ ), identity (59) and scalar products (70),(71). The second term in (83) gives

$$
\ll\left(\lambda \gamma^{a} \theta\right)\left(\theta \gamma^{a} \widetilde{\psi}^{*}\right)\left(\lambda \gamma^{\mu} \theta\right)\left(\lambda \gamma^{\nu} \theta\right)\left(\varepsilon \gamma^{\mu \nu \rho} \theta\right) \gg=\frac{1}{96 \cdot 120}\left(\widetilde{\psi}^{*} \gamma^{a} \gamma_{a \mu \nu} \gamma^{\mu \nu \rho} \varepsilon\right)=-\frac{1}{20}\left(\varepsilon \gamma^{\rho} \widetilde{\psi}^{*}\right)
$$

Collecting together

$$
\mathcal{D}=-20 \cdot \frac{1}{40}\left(\varepsilon \gamma^{\rho} \widetilde{\psi}^{*}\right) A_{\rho}^{*}-20 \cdot \frac{1}{20}\left(\varepsilon \gamma^{\rho} \widetilde{\psi}^{*}\right) A_{\rho}^{*}=-\frac{3}{2}\left(\varepsilon \gamma^{\rho} \widetilde{\psi}^{*}\right) A_{\rho}^{*}
$$

Even from this calculation it is clear that the $Z_{2}$ duality discussed in section 5 looks highly non-trivial at the level of Feynman diagrams. Calculation of the diagram in fig. 7 is considerably simpler than that in the fig. 8 . However, the final result after the identification (55) is the same. In the next set of diagrams we will see more dramatic realization of this duality. Calculation from the one side of this duality looks very simple, calculation from the other side requires a lot of $\gamma$-matrix algebra.

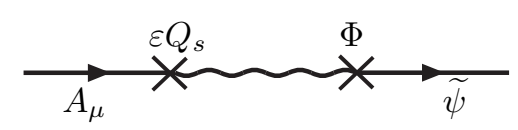

Application of sypersymmetry operator ${ }^{7}$ gives $Q$-exact expression

$$
\varepsilon Q_{s}\left[\left(\lambda \gamma^{\mu} \theta\right) A_{\mu}\right]=\left(\lambda \gamma^{\mu} \varepsilon\right) A_{\mu} .
$$

The pre-image is $\left(\theta \gamma^{\mu} \varepsilon\right) A_{\mu}$. The whole contribution gives

$$
\mathcal{D}=-16 \ll\left(\lambda \gamma^{a} \theta\right)\left(\lambda \gamma^{b} \theta\right)\left(\theta \gamma^{a b} \widetilde{\psi}\right)\left(\lambda \gamma^{\nu} \theta\right)\left(\theta \gamma^{\mu} \varepsilon\right) \gg \partial_{\nu} A_{\mu}=-\frac{16}{96 \cdot 120}\left(\widetilde{\psi} \gamma^{a b} \gamma_{a b \nu} \gamma^{\mu} \varepsilon\right) \partial_{\nu} A_{\mu}=\frac{1}{10}\left(\varepsilon \gamma^{\mu} \gamma^{\nu} \widetilde{\psi}\right) \partial_{\nu} A_{\mu}
$$




$$
\begin{gathered}
\underset{\psi^{*}}{\stackrel{\varepsilon Q_{s}}{x}} \underset{\widetilde{A}_{\mu}^{*}}{\Phi} \\
\varepsilon Q_{s}\left[-16\left(\lambda \gamma^{\mu} \theta\right)\left(\lambda \gamma^{\nu} \theta\right)\left(\theta \gamma^{\mu \nu} \psi^{*}\right)\right]=-2 \cdot 16\left(\lambda \gamma^{\mu} \varepsilon\right)\left(\lambda \gamma^{\nu} \theta\right)\left(\theta \gamma^{\mu \nu} \psi^{*}\right)-16\left(\lambda \gamma^{\mu} \theta\right)\left(\lambda \gamma^{\nu} \theta\right)\left(\varepsilon \gamma^{\mu \nu} \psi^{*}\right)
\end{gathered}
$$

The second term is proportional to that one which appeared in the calculation of kinetic term for the gauge field, and according to (78)

$$
\Pi_{2}=-4\left(\theta \gamma^{a \mu \nu} \theta\right)\left(\lambda \gamma_{a} \theta\right)\left(\varepsilon \gamma^{\mu \nu} \psi^{*}\right)
$$

To write the first term in the convenient form one should expand $\gamma^{\mu \nu}=g^{\mu \nu}-\gamma^{\nu} \gamma^{\mu}$ and use (64)

$$
\begin{gathered}
\Pi_{1}=32\left(\theta \gamma^{\mu} \varepsilon\right)\left(\lambda \gamma^{\nu} \theta\right)\left(\theta \gamma^{\nu} \gamma^{\mu} \psi^{*}\right) \\
\Phi\left(\Pi_{1}+\Pi_{2}\right)=32\left(\lambda \gamma^{\rho} \theta\right)\left(\theta \gamma^{\mu} \varepsilon\right)\left(\lambda \gamma^{\nu} \theta\right)\left(\theta \gamma^{\nu} \gamma^{\mu} \partial_{\rho} \psi^{*}\right)-4\left(\lambda \gamma^{\rho} \theta\right)\left(\theta \gamma^{a \mu \nu} \theta\right)\left(\lambda \gamma_{a} \theta\right)\left(\varepsilon \gamma^{\mu \nu} \partial_{\rho} \psi^{*}\right) \\
\mathcal{D}=32 \ll\left(\lambda \gamma^{d} \theta\right) \widetilde{A}_{d}^{*}\left(\lambda \gamma^{\rho} \theta\right)\left(\theta \gamma^{\mu} \varepsilon\right)\left(\lambda \gamma^{\nu} \theta\right)\left(\theta \gamma^{\nu} \gamma^{\mu} \partial_{\rho} \psi^{*}\right) \gg-4 \ll\left(\lambda \gamma^{d} \theta\right) \widetilde{A}_{d}^{*}\left(\lambda \gamma^{\rho} \theta\right)\left(\theta \gamma^{a \mu \nu} \theta\right)\left(\lambda \gamma_{a} \theta\right) \gg\left(\varepsilon \gamma^{\mu \nu} \partial_{\rho} \psi^{*}\right)= \\
=-\frac{32}{96 \cdot 120}\left(\varepsilon \gamma^{\mu} \gamma_{d \rho \nu} \gamma^{\nu} \gamma^{\mu} \partial_{\rho} \psi^{*}\right) \widetilde{A}_{d}^{*}-\frac{4}{120} \widetilde{A}_{d}^{*} \delta_{d \rho a}^{a \mu \nu}\left(\varepsilon \gamma^{\mu \nu} \partial_{\rho} \psi^{*}\right)=-\frac{2}{9}\left(\varepsilon \gamma^{\nu \mu} \psi^{*}\right) \partial_{\nu} \widetilde{A}_{\mu}^{*} \quad(88) \\
\underbrace{\left(8 Q_{s}\right.}_{A_{\mu}} \underbrace{(88)}_{\widetilde{\psi}}
\end{gathered}
$$

The first part of the diagram is analogous to the one responsible for the kinetic term in the gauge field (78) $\Pi=\frac{1}{4}\left(\theta \gamma^{a \mu \nu} \theta\right)\left(\lambda \gamma_{a} \theta\right) \partial_{\mu} A_{\nu}$. Applying operator $\varepsilon Q_{s}$ one can come to following contribution

$$
\mathcal{D}=-\frac{16}{2} \ll\left(\lambda \gamma^{b} \theta\right)\left(\lambda \gamma^{c} \theta\right)\left(\theta \gamma^{b c} \widetilde{\psi}\right)\left(\varepsilon \gamma^{a \nu \mu} \theta\right)\left(\lambda \gamma^{a} \theta\right) \gg \partial_{\nu} A_{\mu}-\frac{16}{4} \ll\left(\lambda \gamma^{b} \theta\right)\left(\lambda \gamma^{c} \theta\right)\left(\theta \gamma^{b c} \widetilde{\psi}\right)\left(\theta \gamma^{a \nu \mu} \theta\right)\left(\lambda \gamma^{a} \varepsilon\right) \gg \partial_{\nu} A_{\mu}
$$

Contribution of the first and second terms respectively

$$
\begin{gathered}
\mathcal{D}_{1}=\frac{16}{2 \cdot 96 \cdot 120}\left(\varepsilon \gamma^{a \nu \mu} \gamma_{b c a} \gamma^{b c} \widetilde{\psi}\right) \partial_{\nu} A_{\mu}=\frac{576 \cdot 16}{2 \cdot 96 \cdot 120}\left(\varepsilon \gamma^{\mu \nu} \widetilde{\psi}\right) \partial_{\nu} A_{\mu}=\frac{2}{5}\left(\varepsilon \gamma^{\mu \nu} \widetilde{\psi}\right) \partial_{\nu} A_{\mu} \\
\mathcal{D}_{2}=\frac{16}{4 \cdot 2 \cdot 96 \cdot 120}\left(\widetilde{\psi} \gamma^{b c} \gamma_{b c a} \gamma^{\mu \nu} \gamma^{a} \varepsilon\right) \partial_{\nu} A_{\mu}-\frac{16}{4 \cdot 24 \cdot 96 \cdot 70} \delta_{[b}^{[a} \eta_{c][k} \delta_{l}^{q} \delta_{p]}^{r]}\left(\widetilde{\psi} \gamma^{b c} \gamma_{k l p} \gamma^{\mu \nu} \gamma_{a q r} \varepsilon\right) \partial_{\nu} A_{\mu}=\frac{1}{15}\left(\varepsilon \gamma^{\mu \nu} \widetilde{\psi}\right) \partial_{\nu} A_{\mu}
\end{gathered}
$$

Finally

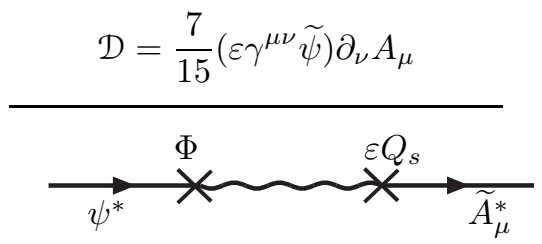

Applying operator $\Phi$ and taking the pre-image one obtains

$$
\Pi=4\left(\theta \gamma^{a \rho \nu} \theta\right)\left(\lambda \gamma_{a} \theta\right)\left(\lambda \gamma^{\mu} \theta\right)\left(\theta \gamma^{\mu} \gamma^{\nu} \partial_{\rho} \psi^{*}\right)
$$

Application of $\varepsilon Q_{s}$ gives 4 terms, corresponding contributions are

$$
\begin{gathered}
\mathcal{D}_{1}=8 \widetilde{A}_{b}^{*} \ll\left(\lambda \gamma^{b} \theta\right)\left(\varepsilon \gamma^{a \rho \nu} \theta\right)\left(\lambda \gamma_{a} \theta\right)\left(\lambda \gamma^{\mu} \theta\right)\left(\theta \gamma^{\mu} \gamma^{\nu} \partial_{\rho} \psi^{*}\right) \gg=\frac{2}{5}\left(\varepsilon \partial_{\rho} \psi^{*}\right) \widetilde{A}_{\rho}^{*}-\frac{4}{15}\left(\varepsilon \gamma^{b \rho} \partial_{\rho} \psi^{*}\right) \widetilde{A}_{b}^{*} \\
\mathcal{D}_{2}=4 \widetilde{A}_{b}^{*} \ll\left(\lambda \gamma^{b} \theta\right)\left(\theta \gamma^{a \rho \nu} \theta\right)\left(\lambda \gamma_{a} \varepsilon\right)\left(\lambda \gamma^{\mu} \theta\right)\left(\theta \gamma^{\mu} \gamma^{\nu} \partial_{\rho} \psi^{*}\right) \gg=\frac{1}{10}\left(\varepsilon \partial_{\rho} \psi^{*}\right) \widetilde{A}_{\rho}^{*}-\frac{1}{30}\left(\varepsilon \gamma^{b \rho} \partial_{\rho} \psi^{*}\right) \widetilde{A}_{b}^{*} \\
\mathcal{D}_{3}=-4 \widetilde{A}_{b}^{*} \ll\left(\lambda \gamma^{b} \theta\right)\left(\theta \gamma^{a \rho \nu} \theta\right)\left(\lambda \gamma_{a} \theta\right)\left(\lambda \gamma^{\mu} \varepsilon\right)\left(\theta \gamma^{\mu} \gamma^{\nu} \partial_{\rho} \psi^{*}\right) \gg=-\frac{1}{5}\left(\varepsilon \partial_{\rho} \psi^{*}\right) \widetilde{A}_{\rho}^{*} \\
\mathcal{D}_{4}=4 \widetilde{A}_{b}^{*} \ll\left(\lambda \gamma^{b} \theta\right)\left(\theta \gamma^{a \rho \nu} \theta\right)\left(\lambda \gamma_{a} \theta\right)\left(\lambda \gamma^{\mu} \theta\right)\left(\varepsilon \gamma^{\mu} \gamma^{\nu} \partial_{\rho} \psi^{*}\right) \gg=-\frac{2}{5}\left(\varepsilon \partial_{\rho} \psi^{*}\right) \widetilde{A}_{\rho}^{*}-\frac{2}{45}\left(\varepsilon \gamma^{b \rho} \partial_{\rho} \psi^{*}\right) \widetilde{A}_{b}^{*}
\end{gathered}
$$

The total contribution is

$$
\mathcal{D}=-\frac{1}{10}\left(\varepsilon \partial_{\rho} \psi^{*}\right) \widetilde{A}_{\rho}^{*}-\frac{31}{90}\left(\varepsilon \gamma^{\mu \nu} \psi^{*}\right) \partial_{\mu} \widetilde{A}_{\nu}^{*}
$$

Again we emphasize that the $Z_{2}$ duality after the identification (55) implies the diagram identity 

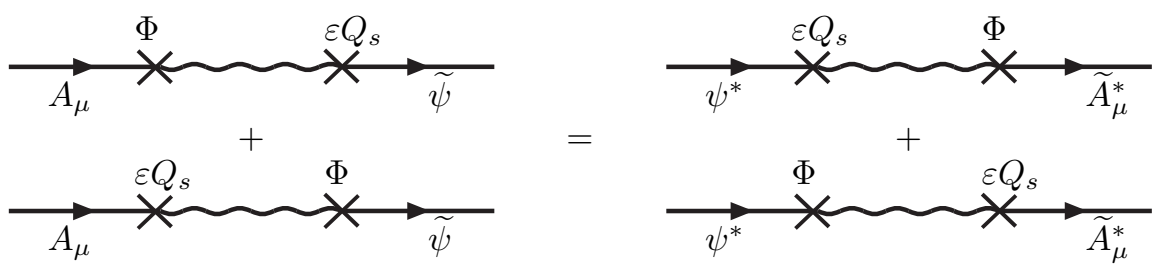

Which is rather non-trivial!

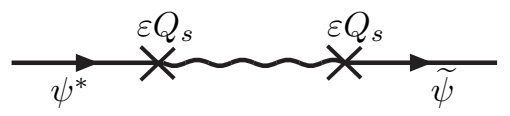

The calculation of the first part of this diagram is analogous to (86),(87). Application of the second operator $\varepsilon Q_{s}\left(\Pi_{1}+\Pi_{2}\right)$ gives 5 terms.

$$
\begin{gathered}
\mathcal{D}_{1}=-2 \cdot 16^{2} \ll\left(\lambda \gamma^{d} \theta\right)\left(\lambda \gamma^{k} \theta\right)\left(\theta \gamma^{d k} \widetilde{\psi}\right)\left(\varepsilon \gamma^{\mu} \varepsilon\right)\left(\lambda \gamma^{\nu} \theta\right)\left(\theta \gamma^{\nu} \gamma^{\mu} \psi^{*}\right) \gg=32\left(\varepsilon \gamma^{\mu} \varepsilon\right)\left(\widetilde{\psi} \gamma^{\mu} \psi^{*}\right) \\
\mathcal{D}_{2}=2 \cdot 16^{2} \ll\left(\lambda \gamma^{d} \theta\right)\left(\lambda \gamma^{k} \theta\right)\left(\theta \gamma^{d k} \widetilde{\psi}\left(\theta \gamma^{\mu} \varepsilon\right)\left(\lambda \gamma^{\nu} \varepsilon\right)\left(\theta \gamma^{\nu} \gamma^{\mu} \psi^{*}\right) \gg=-\frac{8}{5}\left(\widetilde{\psi} \gamma^{a} \gamma^{\mu} \varepsilon\right)\left(\varepsilon \gamma^{a} \gamma^{\mu} \psi^{*}\right)+\right. \\
+\frac{1}{45}\left(\widetilde{\psi} \gamma^{a b c \mu} \varepsilon\right)\left(\varepsilon \gamma^{a b c \mu} \psi^{*}\right)+\frac{8}{15}\left(\widetilde{\psi} g^{b c} \varepsilon\right)\left(\varepsilon \gamma^{b c} \psi^{*}\right) \\
\mathcal{D}_{3}=-2 \cdot 16^{2} \ll\left(\lambda \gamma^{d} \theta\right)\left(\lambda \gamma^{k} \theta\right)\left(\theta \gamma^{d k} \widetilde{\psi}\right)\left(\theta \gamma^{\mu} \varepsilon\right)\left(\lambda \gamma^{\nu} \theta\right)\left(\varepsilon \gamma^{\nu} \gamma^{\mu} \psi^{*}\right) \gg=-\frac{16}{5}\left(\widetilde{\psi} \gamma^{a} \gamma^{\mu} \varepsilon\right)\left(\varepsilon \gamma^{a} \gamma^{\mu} \psi^{*}\right) \\
\mathcal{D}_{4}=\frac{1}{2} \cdot 16^{2} \ll\left(\lambda \gamma^{d} \theta\right)\left(\lambda \gamma^{k} \theta\right)\left(\theta \gamma^{d k} \widetilde{\psi}\right)\left(\varepsilon \gamma^{a \mu \nu} \theta\right)\left(\lambda \gamma_{a} \theta\right)\left(\varepsilon \gamma^{\mu \nu} \psi^{*}\right) \gg=-\frac{32}{5}\left(\widetilde{\psi} \gamma^{b c} \varepsilon\right)\left(\varepsilon \gamma^{b c} \psi^{*}\right) \\
\mathcal{D}_{5}=\frac{1}{4} \cdot 16^{2} \ll\left(\lambda \gamma^{d} \theta\right)\left(\lambda \gamma^{k} \theta\right)\left(\theta \gamma^{d k} \widetilde{\psi}\right)\left(\theta \gamma^{a \mu \nu} \theta\right)\left(\lambda \gamma_{a} \varepsilon\right)\left(\varepsilon \gamma^{\mu \nu} \psi^{*}\right) \gg=-\frac{16}{15}\left(\widetilde{\psi} \gamma^{b c} \varepsilon\right)\left(\varepsilon \gamma^{b c} \psi^{*}\right)
\end{gathered}
$$

Summing up all the contributions and using the identities (63) and (69) one can come to

$$
\mathcal{D}=80\left(\varepsilon \gamma^{\mu} \varepsilon\right)\left(\widetilde{\psi} \gamma^{\mu} \psi^{*}\right)-160(\varepsilon \widetilde{\psi})\left(\varepsilon \psi^{*}\right)
$$

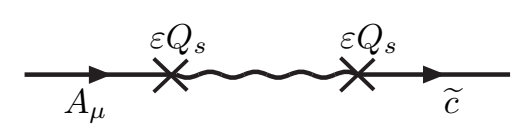

Applying $\varepsilon Q_{s}$ one obtains an exact expression.

$$
\varepsilon Q_{s}\left[\left(\lambda \gamma^{\mu} \theta\right) A_{\mu}\right]=\left(\lambda \gamma^{\mu} \varepsilon\right) A_{\mu}, \Longrightarrow \Pi=\left(\theta \gamma^{\mu} \varepsilon\right) A_{\mu} .
$$

The whole contribution is

$$
\mathcal{D}=\tilde{c} \ll\left(\lambda \gamma^{\mu} \theta\right)\left(\lambda \gamma^{\nu} \theta\right)\left(\lambda \gamma^{\rho} \theta\right)\left(\theta \gamma_{\mu \nu \rho} \theta\right)\left(\varepsilon \gamma^{a} \varepsilon\right) A_{a} \gg=\widetilde{c}\left(\varepsilon \gamma^{\mu} \varepsilon\right) A_{\mu}
$$

The calculation of dual diagram is much more tricky. Operator $Q_{s}$ acts as $\varepsilon \frac{\partial}{\partial \theta}$ and gives 2 terms

$$
\begin{array}{r}
\varepsilon \frac{\partial}{\partial \theta}\left[\left(\lambda \gamma^{\mu} \theta\right)\left(\lambda \gamma^{\nu} \theta\right)\left(\lambda \gamma^{\rho} \theta\right)\left(\theta \gamma^{\mu \nu \rho} \theta\right) c^{*}\right]= \\
3\left(\lambda \gamma^{\mu} \varepsilon\right)\left(\lambda \gamma^{\nu} \theta\right)\left(\lambda \gamma^{\rho} \theta\right)\left(\theta \gamma^{\mu \nu \rho} \theta\right) c^{*}-2\left(\lambda \gamma^{\mu} \theta\right)\left(\lambda \gamma^{\nu} \theta\right)\left(\lambda \gamma^{\rho} \theta\right)\left(\varepsilon \gamma^{\mu \nu \rho} \theta\right) c^{*},
\end{array}
$$

next step

$$
\begin{array}{r}
\Pi_{1}=3\left(\theta \gamma^{\mu} \varepsilon\right)\left(\lambda \gamma^{\nu} \theta\right)\left(\lambda \gamma^{\rho} \theta\right)\left(\theta \gamma^{\mu \nu \rho} \theta\right) c^{*} \\
\Pi_{2}=-\frac{1}{2}\left(\theta \gamma^{\mu \nu \sigma} \theta\right)\left(\lambda \gamma_{\sigma} \theta\right)\left(\lambda \gamma^{\rho} \theta\right)\left(\varepsilon \gamma^{\mu} \gamma^{\nu} \gamma^{\rho} \theta\right) c^{*}
\end{array}
$$


After applying second $\varepsilon Q_{s}$ there arise seven terms

$$
\begin{array}{r}
\varepsilon Q_{s}\left(\Pi_{1}+\Pi_{2}\right)=3\left(\varepsilon \gamma^{\mu} \varepsilon\right)\left(\lambda \gamma^{\nu} \theta\right)\left(\lambda \gamma^{\rho} \theta\right)\left(\theta \gamma^{\mu \nu \rho} \theta\right)-6\left(\theta \gamma^{\mu} \varepsilon\right)\left(\lambda \gamma^{\nu} \varepsilon\right)\left(\lambda \gamma^{\rho} \theta\right)\left(\theta \gamma^{\mu \nu \rho} \theta\right) \\
-6\left(\theta \gamma^{\mu} \varepsilon\right)\left(\lambda \gamma^{\nu} \theta\right)\left(\lambda \gamma^{\rho} \theta\right)\left(\varepsilon \gamma^{\mu \nu \rho} \theta\right)-\left(\varepsilon \gamma^{\mu \nu \sigma} \theta\right)\left(\lambda \gamma_{\sigma} \theta\right)\left(\lambda \gamma^{\rho} \theta\right)\left(\varepsilon \gamma^{\mu} \gamma^{\nu} \gamma^{\rho} \theta\right) \\
-\frac{1}{2}\left(\theta \gamma^{\mu \nu \sigma} \theta\right)\left(\lambda \gamma_{\sigma} \varepsilon\right)\left(\lambda \gamma^{\rho} \theta\right)\left(\varepsilon \gamma^{\mu} \gamma^{\nu} \gamma^{\rho} \theta\right)+\frac{1}{2}\left(\theta \gamma^{\mu \nu \sigma} \theta\right)\left(\lambda \gamma_{\sigma} \theta\right)\left(\lambda \gamma^{\rho} \varepsilon\right)\left(\varepsilon \gamma^{\mu} \gamma^{\nu} \gamma^{\rho} \theta\right) \\
-\frac{1}{2}\left(\theta \gamma^{\mu \nu \sigma} \theta\right)\left(\lambda \gamma_{\sigma} \theta\right)\left(\lambda \gamma^{\rho} \theta\right)\left(\varepsilon \gamma^{\mu} \gamma^{\nu} \gamma^{\rho} \varepsilon\right)
\end{array}
$$

Some calculus gives us the following results

$$
\begin{aligned}
\ll 3\left(\varepsilon \gamma^{\mu} \varepsilon\right)\left(\lambda \gamma^{\nu} \theta\right)\left(\lambda \gamma^{\rho} \theta\right)\left(\theta \gamma^{\mu \nu \rho} \theta\right)\left(\lambda \gamma^{a} \theta\right) \gg & =\frac{3}{10}\left(\varepsilon \gamma^{a} \varepsilon\right), \\
-6 \ll\left(\theta \gamma^{\mu} \varepsilon\right)\left(\lambda \gamma^{\nu} \theta\right)\left(\lambda \gamma^{\rho} \theta\right)\left(\varepsilon \gamma^{\mu \nu \rho} \theta\right)\left(\lambda \gamma^{a} \theta\right) \gg & =\frac{9}{40}\left(\varepsilon \gamma_{a} \varepsilon\right), \\
-\ll\left(\varepsilon \gamma^{\mu \nu \sigma} \theta\right)\left(\lambda \gamma_{\sigma} \theta\right)\left(\lambda \gamma^{\rho} \theta\right)\left(\varepsilon \gamma^{\mu} \gamma^{\nu} \gamma^{\rho} \theta\right)\left(\lambda \gamma^{a} \theta\right) \gg & =\frac{1}{4}\left(\varepsilon \gamma_{a} \varepsilon\right), \\
-\frac{1}{2} \ll\left(\theta \gamma^{\mu \nu \sigma} \theta\right)\left(\lambda \gamma_{\sigma} \theta\right)\left(\lambda \gamma^{\rho} \theta\right)\left(\varepsilon \gamma^{\mu} \gamma^{\nu} \gamma^{\rho} \varepsilon\right)\left(\lambda \gamma^{a} \theta\right) \gg & =\frac{1}{10}\left(\varepsilon \gamma^{a} \varepsilon\right), \\
-6 \ll\left(\theta \gamma^{\mu} \varepsilon\right)\left(\lambda \gamma^{\nu} \varepsilon\right)\left(\lambda \gamma^{\rho} \theta\right)\left(\theta \gamma^{\mu \nu \rho} \theta\right)\left(\lambda \gamma^{a} \theta\right) \gg & =\left(\frac{3}{32}-\frac{3}{160}\right)\left(\varepsilon \gamma^{a} \varepsilon\right), \\
-\frac{1}{2} \ll\left(\theta \gamma^{\mu \nu \sigma} \theta\right)\left(\lambda \gamma_{\sigma} \varepsilon\right)\left(\lambda \gamma^{\rho} \theta\right)\left(\varepsilon \gamma^{\mu} \gamma^{\nu} \gamma^{\rho} \theta\right)\left(\lambda \gamma^{a} \theta\right) \gg & =\left(\frac{13}{320}-\frac{1}{64}\right)\left(\varepsilon \gamma^{a} \varepsilon\right), \\
\frac{1}{2} \ll\left(\theta \gamma^{\mu \nu \sigma} \theta\right)\left(\lambda \gamma_{\sigma} \theta\right)\left(\lambda \gamma^{\rho} \varepsilon\right)\left(\varepsilon \gamma^{\mu} \gamma^{\nu} \gamma^{\rho} \theta\right)\left(\lambda \gamma^{a} \theta\right) \gg & =\left(\frac{1}{20}-\frac{1}{40}\right)\left(\varepsilon \gamma^{a} \varepsilon\right) .
\end{aligned}
$$

Finally we obtain the same result as in (92)

$$
\mathcal{D}=c^{*}\left(\varepsilon \gamma^{\mu} \varepsilon\right) \widetilde{A}_{\mu}^{*}
$$
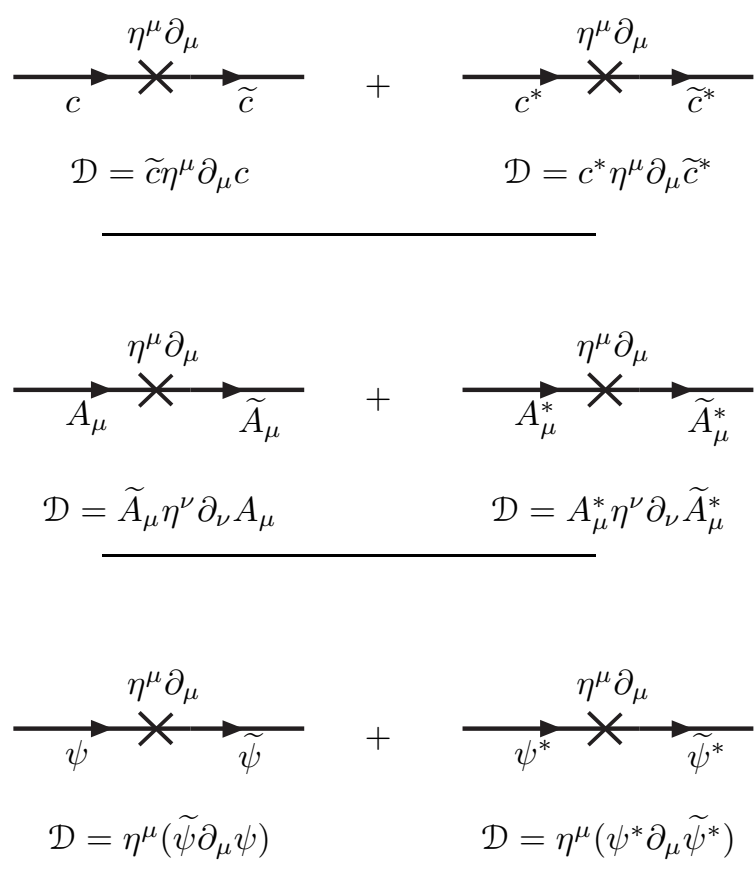

\section{Interaction terms}

The next step is to calculate the contributions proportional to the gauge coupling constant $g$. In contrast to the model discussed in section 4 , the only effect of this interaction is the replacement of operator insertion $\Phi=\left(\lambda \gamma^{\mu} \theta\right) \partial_{\mu}$ by the gauge field $\left(\lambda \gamma^{\mu} \theta\right) A_{\mu}$. In this calculation it is important to remember that all possible permutations of external legs must be considered to obtain the correct gauge invariant result for the effective action. For example, consider the diagram

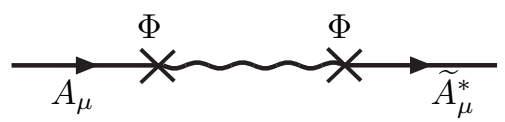


one should add five diagrams

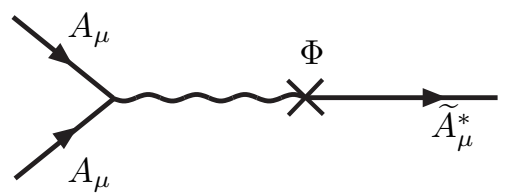

$$
\mathcal{D}=g \frac{1}{360} \widetilde{A}_{\mu}^{*} \partial_{\nu}\left[A_{\mu}, A_{\nu}\right]
$$
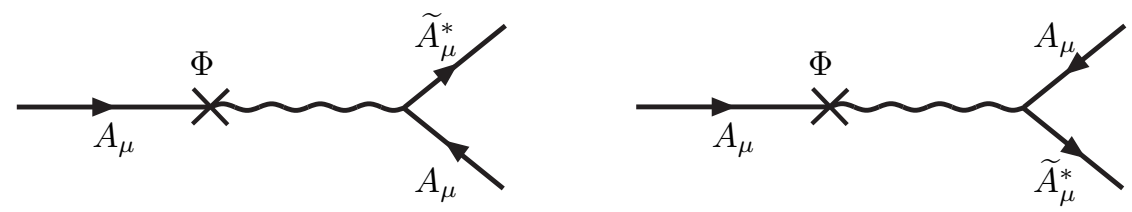

$$
\mathcal{D}=g \frac{1}{360}\left[\widetilde{A}_{\mu}^{*} A_{\nu}\right]\left(\partial_{\mu} A_{\nu}-\partial_{\nu} A_{\mu}\right)
$$
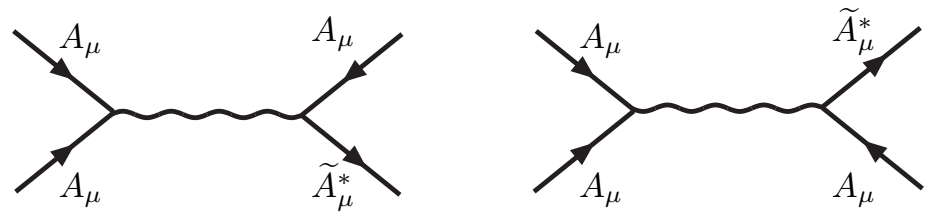

$$
\mathcal{D}=g^{2} \frac{1}{360}\left[\widetilde{A}_{\mu}^{*} A_{\nu}\right]\left[A_{\mu}, A_{\nu}\right]
$$

which are different due to the clockwise rule (see section 5 of [2]).

There is also a subset of trivial diagrams with ghosts. These diagrams are depicted below.
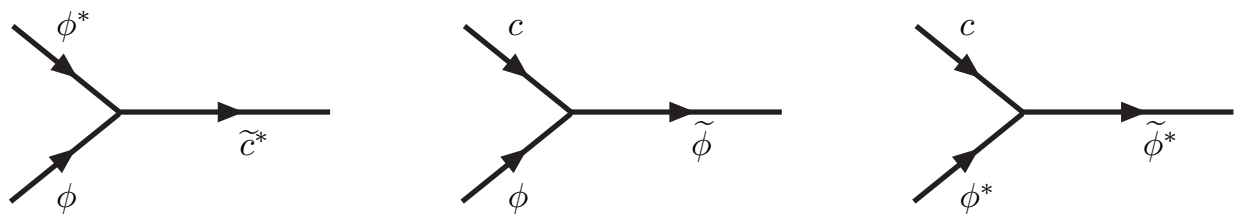

Here $\phi$ stand for arbitrary field $\left(c, A_{\mu}, \psi\right)$. It is important to remember that all the permutations of external legs should be taken into account according to the clockwise rule.

Collecting together the results for all the diagrams one can come to the following result for the effective lagrangian.

$$
\begin{array}{r}
L_{p r e}^{e f f}=-\frac{1}{360} \widetilde{A}_{\mu}^{*} D_{\nu} F_{\mu \nu}+\frac{1}{160} \psi \gamma^{\mu} D_{\mu} \widetilde{\psi}^{*}+\frac{g}{160} \widetilde{A}_{\mu}^{*}\left(\psi \gamma^{\mu} \psi\right)+\widetilde{A}_{\mu} D_{\mu} c+A_{\mu}^{*} D_{\mu} \widetilde{c}^{*}-g A_{\mu}^{*}\left[\widetilde{A}_{\mu}^{*}, c\right]+ \\
+g\left(\widetilde{c} c c+\widetilde{c}^{*}\left[c^{*}, c\right]+\left[\widetilde{\psi}^{*}, \psi^{*}\right] c+[\widetilde{\psi}, \psi] c+\widetilde{c}^{*}\left[\psi^{*}, \psi\right]\right)+ \\
+\frac{3}{2}\left(\varepsilon \gamma^{\mu} \psi\right) \widetilde{A}_{\mu}+\frac{3}{2}\left(\varepsilon \gamma^{\mu} \widetilde{\psi}^{*}\right) A_{\mu}^{*}+\frac{2}{3}\left(\varepsilon \gamma^{\mu \nu} \widetilde{\psi}\right) D_{\mu} A_{\nu}+\frac{2}{3}\left(\varepsilon \gamma^{\mu \nu} \psi^{*}\right) D_{\mu} \widetilde{A}_{\nu}^{*}-\frac{g}{3}\left(\varepsilon \gamma^{\mu \nu} \widetilde{\psi}\right)\left[A_{\mu}, A_{\nu}\right]+ \\
+\eta^{\mu}\left[\widetilde{c} \partial_{\mu} c-c^{*} \partial_{\mu} \widetilde{c}^{*}+\widetilde{A}_{\nu} \partial_{\mu} A_{\nu}-A_{\nu}^{*} \partial_{\mu} \widetilde{A}_{\nu}^{*}+\left(\widetilde{\psi} \partial_{\mu} \psi\right)-\left(\psi^{*} \partial_{\mu} \widetilde{\psi}^{*}\right)\right]-\eta_{\mu}^{*}\left(\varepsilon \gamma^{\mu} \varepsilon\right)- \\
-80\left(\varepsilon \gamma^{\mu} \varepsilon\right)\left(\widetilde{\psi} \gamma_{\mu} \psi^{*}\right)+160(\varepsilon \widetilde{\psi})\left(\varepsilon \psi^{*}\right)-c^{*}\left(\varepsilon \gamma^{\mu} \varepsilon\right) \widetilde{A}_{\mu}^{*}-\widetilde{c}\left(\varepsilon \gamma^{\mu} \varepsilon\right) A_{\mu}
\end{array}
$$

Here $D_{\mu}=\partial_{\mu}+g\left[A_{\mu}, \cdot\right], F_{\mu \nu}=\partial_{\mu} A_{\nu}-\partial_{\nu} A_{\mu}+g\left[A_{\mu}, A_{\nu}\right]$. 


\section{References}

[1] I. A. Batalin and G. A. Vilkovisky, "Gauge Algebra And Quantization," Phys. Lett. B 102 (1981) 27.

I. A. Batalin and G. A. Vilkovisky, "Quantization Of Gauge Theories With Linearly Dependent Generators," Phys. Rev. D 28 (1983) 2567 [Erratum-ibid. D 30 (1984) 508].

I. A. Batalin and E. S. Fradkin, "A Generalized Canonical Formalism And Quantization Of Reducible Gauge Theories," Phys. Lett. B 122 (1983) 157.

B. L. Voronov and I. V. Tyutin, "Formulation Of Gauge Theories Of General Form. I," Theor. Math. Phys. 50 (1982) 218 [Teor. Mat. Fiz. 50 (1982) 333].

[2] D. Krotov, A. Losev, "Quantum field theory as effective BV theory from Chern-Simons", [arXiv:hepth/0603201].

[3] N. Berkovits, "Covariant quantization of the superparticle using pure spinors," JHEP 0109 (2001) 016 [arXiv:hep-th/0105050].

N. Berkovits, "Super-Poincare covariant quantization of the superstring," JHEP 0004 (2000) 018 [arXiv:hep-th/0001035].

N. Berkovits, "ICTP lectures on covariant quantization of the superstring," arXiv:hep-th/0209059.

[4] A. Schwarz, "Geometry of Batalin-Vilkovisky quantization," Commun. Math. Phys. 155 (1993) 249 [arXiv:hep-th/9205088].

A. Schwarz, "Semiclassical approximation in Batalin-Vilkovisky formalism," Commun. Math. Phys. 158 (1993) 373 [arXiv:hep-th/9210115].

[5] M. Movshev and A. Schwarz, "On maximally supersymmetric Yang-Mills theories," Nucl. Phys. B 681, 324 (2004) [arXiv:hep-th/0311132].

M. Movshev and A. Schwarz, "Algebraic structure of Yang-Mills theory," arXiv:hep-th/0404183.

[6] G. Barnich, M. Grigoriev, A. Semikhatov and I. Tipunin, "Parent field theory and unfolding in BRST first-quantized terms," Commun. Math. Phys. 260 (2005) 147 [arXiv:hep-th/0406192].

G. Barnich and M. Grigoriev, "Parent form for higher spin fields on anti-de Sitter space," arXiv:hepth/0602166.

M. Grigoriev, "Off-shell gauge fields from BRST quantization," arXiv:hep-th/0605089.

[7] E. S. Fradkin and M. A. Vasiliev, "Hamiltonian Formalism, Quantization and S Matrix for Supergravity," Phys. Lett. B 72 (1977) 70.

M. B. Green and C. M. Hull, "The covariant quantization of the superparticle," QMC/PH/89-7. Presented at Texas A and M Mtg. on String Theory, College Station, TX, Mar 13-18, 1989

M. B. Green and C. M. Hull, "Quantum Mechanics of a Twisted Ssuperparticle," Nucl. Phys. B 344, 115 (1990).

M. B. Green and C. M. Hull, "The quantum mechanics of an $n+1$ superparticle in an extended superspace," Mod. Phys. Lett. A 5, 1399 (1990).

E. Bergshoeff, R. Kallosh and A. Van Proeyen, "Superparticle Actions And Gauge Fixings," Class. Quant. Grav. 9, 321 (1992).

R. E. Kallosh, "Covariant quantization of type iia, iib green-schwarz superstring," Phys. Lett. B 225, 49 (1989).

[8] P. Mnev, "Notes on simplicial BF theory," arXiv:hep-th/0610326.

V. Dolotin, A. Morozov and S. Shakirov, "A-infinity structure on simplicial complexes," arXiv:0704.2609 [math.GT].

[9] A. Bilal, "Introduction to Supersymmetry", arXiv:hep-th/0101055.

[10] P. A. Grassi, G. Policastro and E. Scheidegger, "Partition functions, localization, and the chiral de Rham complex," arXiv:hep-th/0702044. 
[11] N. Berkovits and C. R. Mafra, "Some superstring amplitude computations with the non-minimal pure spinor formalism," arXiv:hep-th/0607187.

[12] U. Gran, "GAMMA: A Mathematica package for performing Gamma-matrix algebra and Fierz transformations in arbitrary dimensions," arXiv:hep-th/0105086.

[13] N. Berkovits and N. Nekrasov, "Multiloop superstring amplitudes from non-minimal pure spinor formalism," arXiv:hep-th/0609012.

[14] A. L. Gorodentsev and A. N. Rudakov, "On syzygies of highest weight orbits," arXiv:math.ag/0602316.

[15] M. Cederwall, B. E. W. Nilsson and D. Tsimpis, "Spinorial cohomology and maximally supersymmetric theories," JHEP 0202 (2002) 009 [arXiv:hep-th/0110069].

M. Cederwall, B. E. W. Nilsson and D. Tsimpis, "D = 10 super-Yang-Mills at O(alpha**2)," JHEP $\mathbf{0 1 0 7}$ (2001) 042 [arXiv:hep-th/0104236].

M. Cederwall, B. E. W. Nilsson and D. Tsimpis, "The structure of maximally supersymmetric Yang-Mills theory: Constraining higher-order corrections," JHEP 0106 (2001) 034 [arXiv:hep-th/0102009].

[16] A. Galperin, E. Ivanov, S. Kalitsyn, V. Ogievetsky and E. Sokatchev, "Unconstrained N=2 Matter, YangMills And Supergravity Theories In Harmonic Superspace," Class. Quant. Grav. 1 (1984) 469.

E. Nissimov, S. Pacheva and S. Solomon, "Off-shell superspace D = 10 super-Yang-Mills from covariantly quantized Green-Schwarz superstring," Nucl. Phys. B 317 (1989) 344.

E. Nissimov, S. Pacheva and S. Solomon, "The relation between operator and path integral covariant quantizations of the Green-Schwarz superstring," Phys. Lett. B 228 (1989) 181.

[17] M. F. Sohnius, "Introducing Supersymmetry," Phys. Rept. 128 (1985) 39. 